\title{
$\mathrm{C} \cdot \mathrm{A} \cdot \mathrm{P}$ Analyse
}

Bettina Thalmaier

\section{Möglichkeiten und Grenzen einer europäischen \\ Identitätspolitik}

Dr. Bettina Thalmaier ist wissenschaftliche Mitarbeiterin in der Bertelsmann Forschungsgruppe Politik am Centrum für angewandte Politikforschung (C.A.P). Der Beitrag ist in leicht abgeänderter Form dem Buch „Europäische Identität: Voraussetzungen und Strategien“ entnommen, das Anfang 2007 in der Reihe „Münchner Beiträge zur europäischen Einigung“ (hrsg. von Werner Weidenfeld und Julian Nida-Rümelin) erscheinen wird. 



\section{$\mathrm{C} \cdot \mathrm{A} \cdot \mathrm{P}$}

\section{Inhalt}

1. Identität als Legitimationsfaktor

2. Gründe für die nur schwach ausgeprägte europäische Identität 7

2.1. Andersartigkeit der EU

2.2. Beharrungsvermögen des nationalstaatlichen Prinzips

2.3. Institutionelles Demokratiedefizit und die nur schwach ausgeprägte europäische Öffentlichkeit

3. Möglichkeiten europäischer identitätsstiftender Politik

3.1. Eindämmung von Systemdynamik und -komplexität

3.2. Abschwächung der Geltung des nationalstaatlichen Prinzips 14

3.3. Mehr Partizipation

3.4. Politisierung europäischer Politik

3.4.1. Institutionelle Reformen

3.4.2. Mehr Dialog und Debatte

4. Fazit 


\section{$\mathrm{C} \cdot \mathrm{A} \cdot \mathrm{P}$}

\section{Möglichkeiten und Grenzen einer europäischen Identitätspolitik}

\section{Zusammenfassung}

Zwar verfolgt die EU bereits seit mehreren Jahrzehnten eine identitätsstiftende Politik. Bislang haben diese Bemühungen zur Konstruktion einer europäischen Identität jedoch nicht dazu geführt, dass sich ein europäisches „Wir-Gefühl“ mit einem annähernd vergleichbaren Dichtegrad wie bei den nationalen Identitäten entwickelt hat. Bei allen vergangenen und zukünftigen Versuchen zur Stärkung der europäischen Identität sehen sich die politischen Akteure mit mehreren spezifischen Gründen konfrontiert, die eine ausgeprägte Identifikation mit der EU erschweren. Zum einen weist die institutionelle Struktur der Union seit ihrer Gründung gemessen an nationalstaatlichen Standards ein demokratisches Defizit auf. Neben diesem institutionellen Demokratiedefizit besteht aber auch ein strukturelles Demokratiedefizit, das sich insbesondere im Mangel intermediärer Vermittlerstrukturen (Medien, Parteien, Verbände etc.) sowie in einer nur schwach ausgeprägten Europäisierung nationaler Teilöffentlichkeiten und einer noch schwächeren gesamteuropäischen Öffentlichkeit äußert. Aber auch die Andersartigkeit der EU gegenüber den herkömmlichen internationalen Organisationen einerseits sowie Nationalstaaten andererseits und das Beharrungsvermögen des nationalstaatlichen Prinzips können als Gründe identifiziert werden, warum sich die europäischen Bürger trotz des beinahe 50-jährigen Bestehens der EU mit ihr nicht sehr stark identifizieren.

Darauf aufbauend wird näher untersucht, inwieweit Möglichkeiten, aber auch Grenzen zur Stärkung der europäischen Identität bestehen. Dabei zeigt sich, dass die Bilanz unterschiedlich ausfällt. An manchen Gründen für die nur schwach ausgeprägte europäische Identität wird sich nur schwerlich etwas ändern lassen, bei anderen besteht ein größerer Handlungsspielraum. Angesichts seiner fehlenden Stabilität und Dauerhaftigkeit sowie seiner Komplexität wird sich das politische System der EU auch in Zukunft als wenig identitätsstiftend erweisen. Allenfalls eine Anerkennung des „Baustellencharakters" der EU als positives Strukturmerkmal und eine transparentere Abgrenzung der Kompetenzen zwischen der EU und den Mitgliedstaaten versprechen hier etwas Abhilfe. Strategien zur Stärkung der europäischen Identität im Bereich der nationalen Schul- und Bildungspolitik sowie die Vermittlung einer neuen Begründungslogik für den europäischen Integrationsprozess werden erst langfristig Wirkungen zeigen.

Das größte Potenzial liegt im Bereich institutioneller Änderungen, mit denen eine stärkere Partizipation am europäischen Entscheidungsprozess verbunden ist und die eine Reduzierung des seit langem beklagten Demokratiedefizits beinhalten. Der Ausbau der Partizipationsmöglichkeiten kann außerdem auch einer Ausweitung der Handlungsstrukturen politischer Öffentlichkeit dienen und zu einer Intensivierung der intermediären Vermittlungsstrukturen beitragen. Erst die verstärkte Ausbildung eines europäischen Kommunikationsraums, der den europäischen Bürgern die Wahrnehmung von Gemeinsamkeiten erlaubt, wird bei diesen ein belastbares Gemeinschaftsgefühl als Grundlage für eine stabile europäische Unterstützung des europäischen Projekts generieren. Maßgeblich sind insoweit eine stärkere Politisierung europäischer Politik und der Aufbau einer europäischen "Streitkommunikation“. 


\section{$\mathrm{C} \cdot \mathrm{A} \cdot \mathrm{P}$}

\section{Identität als Legitimationsfaktor}

Die zunehmende politische Bedeutung des Identitätsthemas im europäischen Kontext resultiert aus der allgemeinen Befürchtung, der europäische Einigungsprozess könne am Negativvotum der Bürger scheitern. Die gegenwärtige Orientierungskrise der Europäischen Union (EU), die maßgeblich durch die Erweiterung von 2004 ausgelöst und durch die beiden negativen Referenden in Frankreich und den Niederlanden im Frühsommer 2005 verstärkt worden ist, hat nicht zuletzt mit einer nur diffus ausgebildeten europäischen Identität zu tun. Abnehmende Akzeptanzwerte müssen die EU beunruhigen, da die Union wie jedes (demokratisch verfasste) politische System auf Anerkennung und Legitimation angewiesen ist.

Zwar verfolgt die EU bereits seit mehreren Jahrzehnten eine identitätsstiftende Politik. ${ }^{1}$ Bislang haben diese Bemühungen zur Konstruktion einer europäischen Identität jedoch nicht dazu geführt, dass sich ein europäisches „Wir-Gefühl“ mit einem annähernd vergleichbaren Dichtegrad wie bei den nationalen Identitäten entwickelt hat. In den Europawissenschaften besteht weitgehend Einigkeit, dass die noch schwach ausgeprägte europäische Identität hinter der nationalen Identität deutlich zurücksteht. Bei allen vergangenen und zukünftigen Versuchen zur Stärkung der europäischen Identität sehen sich die politischen Akteure mit mehreren spezifischen Gründen konfrontiert, ${ }^{2}$ die eine ausgeprägte Identifikation mit der EU erschweren. Zum einen weist die institutionelle Struktur der Union seit ihrer Gründung gemessen an nationalstaatlichen Standards ein demokratisches Defizit auf. ${ }^{3}$ Neben diesem institutionellen Demokratiedefizit ${ }^{4}$ besteht aber auch ein strukturelles Demokratiedefizit ${ }^{5}$, das sich insbesondere im Mangel intermediärer Vermittlerstrukturen (Medien, Parteien, Verbände etc.) sowie in einer nur schwach ausgeprägten Europäisierung nationaler Teilöffentlichkeiten und einer noch schwächeren gesamteuropäischen Öffentlichkeit äußert. Aber auch die Andersartigkeit der EU gegenüber den herkömmlichen internationalen Organisationen einerseits sowie Nationalstaaten andererseits und das Beharrungsvermögen des nationalstaatlichen Prinzips können als Gründe identifiziert werden, warum sich die europäischen Bürger trotz des beinahe 50-jährigen Bestehens der EU mit ihr nicht sehr stark identifizieren.

Gleichwohl ist die EU auf eine eigene kollektive Identität angewiesen. Ein politisches Gemeinwesen - und damit auch die EU - wird von seinen Bürgern nur dann (faktisch) akzeptiert und damit legitimiert, wenn sie sich mit diesem identifizieren. Erachten die Bürger die für alle verbindlichen Entscheidungen nicht für legitim, verfügt das Gemeinwesen nur über eine prekäre Existenz. Die faktische Akzeptanz einer politischen Ordnung durch die Bürger ist daher ein wesentlicher Faktor für deren Stabilität. 6 Dabei lassen sich nach Easton zwei Formen von tatsächlicher Anerkennung unterscheiden:7 Die sogenannte spezifische Unterstützung beruht darauf, dass das politische System Politikergebnisse hervorbringt, die den eigenen Interessen der Bürger entsprechen. Die sogenannte diffuse Unterstützung ist hingegen gerade unabhängig von den gegenwärtigen oder für die Zukunft zu erwartenden Leistungen des Systems, bleibt also auch bestehen, wenn die eigenen partikularen Interessen nicht zum Zuge kommen.

Eine einseitige Fokussierung auf die Erlangung von spezifischer Unterstützung lässt befürchten, dass eine zu große Abhängigkeit von erbrachten Politikergebnissen geschaffen wird. Handelt die Politik aus Sicht der Bürger in deren
Gründe für mangelnde Identifikation mit der EU

Bedarf an einer eigenständigen europäischen Identität 


\section{$\mathrm{C} \cdot \mathrm{A} \cdot \mathrm{P}$}

Stärkung der Output- und Input-Legitimität der EU

Kein europäisches „nation building“
Interesse, wird Zustimmung erteilt, andernfalls bleibt sie aus. Nur wenn ein politisches System auch über eine diffuse Unterstützung verfügt, so die Schlussfolgerung, kann ein grundsätzliches Vertrauen in die Institutionen und deren Handeln generiert werden, das unabhängig von konkreten Ergebnissen des politischen Tagesgeschäfts und partikularen Interessenkonstellationen besteht und auch erhalten bleibt. Die EU kann sich daher „nicht allein durch die Politik, die sie [be]treibt, ausreichend (...) legitimieren (...). Kosten-Nutzen-Kalküle sind keine dauerhafte, belastbare Grundlage eines politischen Systems. Die EU braucht nicht nur specific support, sondern auch diffuse Unterstützung". ${ }^{8}$ Auch die Europäische Union sollte daher bestrebt sein, bei den Bürgern eine diffuse Unterstützung zu generieren.

Als Lehre aus den gescheiterten Referenden kommt es entgegen der Ansicht vieler Politiker und Experten, wonach sich die EU nun mehr auf die Inhalte und weniger auf die Strukturen ihrer Politik konzentrieren müsse, da den Bürger in erster Linie interessiere, was ihm die Europäische Union an konkreten Nutzen und Vorteilen bringe, für die Europäische Union nicht allein darauf an, verstärkt Output zu produzieren und eine „Politik der Ergebnisse“ zu verfolgen. In der Tat erfüllt die Union vielfach nicht die Erwartungen der Bürger, insbesondere im Bereich der Wirtschafts- und Sozialpolitik, ${ }^{9}$ was auch daran liegt, dass diese Politikbereiche weitgehend nicht von der EU, sondern von den einzelnen Mitgliedstaaten verantwortet werden. Die Bürger sind sich nicht mehr sicher, ob die Mitgliedschaft in der Europäischen Union ihnen Prosperität und einen hohen Lebensstandard verschafft. Wohlstand ist derzeit das wichtigste öffentliche Gut der EU, und die Unfähigkeit, dies in angemessenem Umfang bereitzustellen, unterminiert die Legitimation der europäischen Institutionen. Eine Stärkung des Outputs europäischer Politik kann jedoch nur ein, wenn auch äußerst wichtiger Schritt sein. ${ }^{10}$ Neben dem Inhalt sind auch die Strukturen europäischer Politik zu verbessern, um die Unterstützung der Bevölkerung für das europäische Projekt auf eine demokratischere und solidere Grundlage zu stellen. ${ }^{11}$

Um eine diffuse Unterstützung der Bürger für ein politisches Gemeinwesen aufzubauen, ist die Herausbildung einer kollektiven Identität und damit die Identifikation der Bürger mit der Gemeinschaft erforderlich. ${ }^{12}$ Die Ausbildung einer europäischen Identität soll allerdings von vornherein nicht die Schaffung einer europäischen Nationalidentität bezwecken. ${ }^{13}$ Bei der Forcierung einer europäischen Identitätsbildung geht es nicht um den Versuch, nach dem Vorbild des modernen Nationalstaates ein europäisches Kollektivbewusstsein zu konstruieren, unabhängig davon, ob dies überhaupt erfolgreich möglich wäre, d.h. die notwendigen gesellschaftspolitischen, machtpolitischen und sozialpsychologischen Voraussetzungen dafür vorliegen würden. Denn das Ziel des Integrationsprozesses ist nicht die Errichtung eines europäischen (National-)Staates, der die Auflösung der Mitgliedstaaten beinhalten würde. Vielmehr ist von einem offenen Integrationsprozess, der vom Charakter der EU als Herrschaftsverband eigener Art bestimmt wird, als Zielvorstellung auszugehen. ${ }^{14}$ Die EU ist nicht als in einem permanenten Übergangszustand befindlich zu begreifen, sondern es ist von einem Szenario auszugehen, das mit "Maastricht als Dauerzustand“ umschrieben wird. 15 Folglich sollen die nationalen Identitäten nicht zu einer "Nation Europa" verschmelzen, die nationalen Identitäten also nicht durch eine europäische Identität ersetzt werden. Die Homogenisierung der europäischen Bürger im ethno-kulturellen oder auch sozio-kulturellen Sinne ist daher gar nicht erforderlich, weil nicht angestrebt. Ein 


\section{$\mathrm{C} \cdot \mathrm{A} \cdot \mathrm{P}$}

europäisches "nation building" ist zudem auch nicht erstrebenswert, da die Konstruktion nationaler Identitäten vor allem durch gewaltsame Abgrenzung nach außen und Unterdrückung nationaler Minderheiten nach innen vonstatten ging. Im Ergebnis ist daher ein vergleichbar hohes Maß an Gemeinsamkeiten und Übereinstimmungen wie bei nationalen Identitäten nicht erforderlich, unabhängig davon, welche Dichte an Gemeinsamkeiten man zu ihrer Verwirklichung verlangt. Geht man davon aus, dass kollektive Identitäten nicht naturwüchsig oder vorhistorisch vorhanden sind, sondern in sozialen Prozessen konstruiert werden, und sich daher auch eine europäische Identität durch die demokratische Praxis herausbilden kann, ${ }^{16}$ so stellt sich die Frage, wie diese Erfolg versprechend befördert werden kann. Im folgenden Beitrag sollen zunächst die Gründe für die nur schwach ausgeprägte Identifikation der Bürger mit der EU näher erläutert und - darauf aufbauend - ausgelotet werden, inwieweit Möglichkeiten, aber auch Grenzen zur Stärkung der europäischen Identität bestehen.

\section{Gründe für die nur schwach ausgeprägte europäische Identität}

\subsection{Andersartigkeit der EU}

Die Einzigartigkeit der EU als Staatenverbindung eigener Art17 ist der spezifischen Gründungslogik des europäischen Integrationsprozesses geschuldet. Ihr offener und dynamischer Charakter erweist sich als positiv, ist doch eine flexible Antwort auf externe Herausforderungen in einem solchen Umfeld grundsätzlich leichter möglich. Dieser Vorteil erweist sich allerdings bei der Frage der Identifikation als Nachteil. So braucht Identität einen festen und vorstellbaren Bezugsrahmen, auf den sie sich beziehen kann. ${ }^{18}$ Bei der Union fehlt aber das klar umrissene und dauerhaft fixierte Identifikationsobjekt. Auch der hohe Grad an systemischer Komplexität erschwert die Identifikation.

Die Einzigartigkeit der EU als „Herrschaftsverband eigener Art" äußert sich vor allem in dem fehlenden Bezugsrahmen der Union. Erstens versteht sich die EU als dynamisches Gebilde, das einem stetigen Wandel unterliegt. ${ }^{19}$ Europa ist kein geographisch eindeutig definierter Raum, der quasi eine natürliche Grenze hat. Angesichts ständiger Erweiterungsrunden handelt es sich bei der EU vielmehr um ein territorial nicht dauerhaft fixiertes Objekt von Identifikation. ${ }^{20}$ Neben dem Bezugsraum unterliegt auch der rechtliche und politische Rahmen einer stetigen Veränderung, wird das europäische Primärrecht doch alle paar Jahre - zum Teil auch grundlegend - geändert. Zentrales Charakteristikum des Integrationsprozesses ist zudem seine unbestimmte Finalität. ${ }^{21}$ Die Entwicklung der EU ist zu keinem Zeitpunkt einem bestimmten Bauplan oder einer bestimmten Integrationstheorie gefolgt, so dass kein vorgegebenes Integrationsziel besteht, das - wenn auch über einen langen Zeitraum - einfach angesteuert werden müsste.

Zweitens sind die Organ- und Entscheidungsstrukturen der EU als sehr komplex und intransparent anzusehen. ${ }^{22}$ Das liegt zum einen an der unüberschaubaren Anzahl an Entscheidungs- und Rechtssetzungsverfahren, aber auch an der Vielzahl der beteiligten Institutionen und Akteure. Das politisch-administrative System der EU ist von einem polyzentrischen Charakter gekennzeichnet, der sich durch ein nicht-hierarchisches System des Neben- und Miteinanders mehr oder weniger gleichrangiger institutioneller Akteure auszeichnet. Es existiert kein Agenda-setzendes Zentrum und keine eindeutige Gewaltenteilung. Stattdessen

Fehlender Bezugsrahmen der EU

Komplexe und intransparente Organ- und Entscheidungsstrukturen 


\section{$\mathrm{C} \cdot \mathrm{A} \cdot \mathrm{P}$}

sind die Beziehungen zwischen den Akteuren von gegenseitigen Abhängigkeiten und Verflechtungen geprägt. Alle wichtigen Entscheidungen werden nur im Verbund der verschiedenen Systemebenen mittels Verhandlungslösungen getroffen. Statt Befehlsmacht oder Mehrheitsentscheid dominiert eine konsensorientierte Entscheidungsfindung. Die Ausgestaltung der institutionellen Ordnung der EU als Politikverflechtungs-, Verhandlungs- und Konkordanzsystem ${ }^{23}$ über verschiedene territoriale und funktionale Ebenen hat zur Folge, dass es an einer klaren Kompetenzabgrenzung zwischen der Union und den Mitgliedstaaten (und auch Regionen) mangelt. Eine eindeutige Zurechenbarkeit von Erwartungen und Verantwortung ist kaum möglich. ${ }^{24}$ Insbesondere die Politikverflechtung zwischen europäischer und nationaler Ebene erlaubt es den Regierungen der Mitgliedstaaten, sich Leistungen der Integration selbst zuzurechen, obgleich es sich um Erfolge auf europäischer Ebene handelt. Aus demselben Grund ist auch leicht, die Verantwortung für das „Versagen“ nationaler Politik auf die europäische Ebene zu schieben, d.h. die EU wird für Dinge haftbar gemacht, für die nicht die Union, sondern eigentlich die nationale Ebene verantwortlich ist.

\subsection{Beharrungsvermögen des nationalstaatlichen Prinzips}

Umfragen zeigen auch 50 Jahre nach dem Beginn des europäischen Einigungsprozesses die anhaltend starke Bindungskraft der Nation und auch teilweise der Region. ${ }^{25}$ Gerade im Zeitalter der Globalisierung ist ein verstärkter Rückzug auf die nationale Scholle zu beobachten. Hierfür lassen sich im Wesentlichen zwei Gründe anführen, nämlich zum einen die besondere Gestalt der Union und zum anderen der Mangel an einem überzeugenden Narrativ für das europäische Integrationsprojekt.

Das spezifische „Wesen“ der EU
Erstens spielt das "Wesen“ der EU eine entscheidende Rolle. Da es sich bei der Union um eine - wenn auch sehr enge - Kooperation souveräner Staaten handelt, gilt der Grundsatz von den Mitgliedstaaten als "Herren der Verträge“, der eine starke Stellung der Mitgliedstaaten innerhalb des institutionellen Systems der EU aufgrund deren rechtlicher - und auch faktischer - Letztentscheidungsbefugnis beinhaltet. Der Nationalstaat ist auch innerhalb der Union der maßgebliche Akteur, hat doch die EU selbst dazu beigetragen, dass gerade durch den Kompetenzverzicht der beteiligten Staaten deren Souveränität gestärkt werden konnte. Mit der EU haben sich die Mitgliedstaaten einen neuen zusätzlichen Handlungsrahmen geschaffen, den sie bewusst für ihre Zwecke nutzen und beeinflussen können. ${ }^{26}$ Der erweiterte Handlungsspielraum ermöglicht es den beteiligten Staaten, ihre Durchsetzungs- und Problemlösungsfähigkeit zu steigern und Ziele zu verfolgen, die ohne Rückendeckung durch das regionale Regime gar nicht erreichbar wären. ${ }^{27}$ Die Union konnte - und kann - die Erosion nationalstaatlicher Steuerungs- und Problemlösungsfähigkeit durch zwischenstaatliche Kooperation signifikant kompensieren. Obgleich die EU damit einen entscheidenden Beitrag dazu leistet, die europäischen Nationalstaaten funktionsfähig zu erhalten, wird diese Leistung im öffentlichen Bewusstsein kaum wahrgenommen.

Die Eigenart der EU kommt auch darin zum Tragen, dass diese über die Jahrzehnte ihres Bestehens hinweg vor allem ein Projekt der Wirtschaftsintegration gewesen ist, das kaum Identität zu stiften vermag, weil alle Bereiche, in denen einen solche Identitätsstiftung stattfinden könnte, wie Außen- und Innenpolitik oder Sozialpolitik, größtenteils auch bis heute nicht der Gemeinschaftsmethode unterworfen 


\section{$\mathrm{C} \cdot \mathrm{A} \cdot \mathrm{P}$}

sind. Zwar verfügt die EU bereits seit ihrer Gründung über eine politische Dimension und die Aufgaben der EU reichen mittlerweile in zentrale Gebiete klassischer Staatsaufgaben hinein. Der Schwerpunkt der Tätigkeit der EU, in der sie zu einer eigenständigen Rechtsetzung befugt ist, liegt aber auch heute noch bei den regulativen Politiken, d.h. der Regelung wirtschaftlicher Sachverhalte im mikroökonomischen Bereich („,low politics“), im Gegensatz zu den redistributiven Politiken. Da Sozial- und Arbeitsmarktpolitik oder Fragen der inneren und äußeren Sicherheit ("high politics") aufgrund der dabei auftretenden Verteilungsfragen und Wertekonflikte wesentlich "politischer" sind, vermögen sie auch eher, eine Identifikation hervorzurufen. Die Kompetenzverteilung zwischen den Mitgliedstaaten und der EU hat aber vor allem auch deswegen einen nicht unerheblichen Anteil am Beharrungsvermögen des Nationalbewusstseins, weil der Bildungs- und Erziehungsbereich - von einzelnen Fragestellungen mit grenzüberschreitendem wirtschaftlichem Charakter abgesehen - in die Zuständigkeiten der Mitgliedstaaten fällt. Identitätsstiftende Politik findet in modernen Gesellschaften aber vor allem über Erziehungs- und Bildungspolitik statt. ${ }^{28}$ So wird das Nationalbewusstsein vorrangig in diesen Bereichen vermittelt und stets neu konstruiert bzw. bestätigt. Dabei reproduzieren die nationalen Bildungssysteme und die nationalen Medien vornehmlich das jeweilige Nationalbewusstsein, nicht aber ein europäisches Bewusstsein. ${ }^{29}$

Zweitens fehlt es nach der Überwindung der Teilung des Kontinents an einem überzeugenden Narrativ für die Europäische Union. Die alten Motive für das europäische Integrationsprojekt, insbesondere die Versöhnung Europas, Sicherheit und Wohlstand in Europa, wirtschaftlicher Wohlstand und Mobilität, wirken zwar weiterhin und sind weiterhin von Bedeutung. Sie haben aber viel von ihrer Überzeugungskraft verloren, weil die ursprünglichen Integrationsziele heute weitgehend gelebte Realität sind. Historisch bedeutsame Leistungen des Integrationsprozesses sind im Bewusstsein vieler Europäer längst konsumiert und zu Selbstverständlichkeiten geworden. Dabei steckt Europa in einer mentalen Orientierungskrise: "Orientierungslosigkeit ist kein europäisches Phänomen, doch es betrifft Europa in besonderem Maße. Im Zeitalter der globalisierten Moderne haben sich weltweit vorgefundene tradierte Interpretationsordnungen aufgelöst. Durch ein bisher beispielloses Maß an Mobilität, Pluralität und Flexibilität sind alte Identifikationsmöglichkeiten zerbrochen. Dem modernen Menschen fehlt es an wirtschaftlicher, gesellschaftlicher und politischer Orientierung. Der Bedarf an gemeinsamen Umweltbeschreibungen und Zuordnungen ist entsprechend hoch. Die Europäische Union als ein unvollendetes politisches System im Werden ist unter diesen Bedingungen besonders gefordert, Antworten auf den Verlust an Orientierung zu bieten." 30 Die Erbringung einer Orientierungsleistung von Seiten der europäischen Ebene wird jedoch durch den allgemeinen Vertrauensverlust der Bürger in die Gestaltungs- und Steuerungsfähigkeit der Politik erschwert. Die zunehmende Skepsis der Bürger gegenüber den eigenen politischen Eliten hat auch - negative - Auswirkungen auf deren Einstellung zur EU. Da die europäische Integration vornehmlich als Projekt des politischen Establishments angesehen wird, wirkt sich nationale Politikverdrossenheit daher auch negativ auf die Union aus.

Fehlendes überzeugendes Narrativ 


\section{$\mathrm{C} \cdot \mathrm{A} \cdot \mathrm{P}$}

\subsection{Institutionelles Demokratiedefizit und die nur schwach ausgeprägte europäische Öffentlichkeit}

Institutionelles

Demokratiedefizit
Öffentlichkeitsdefizit

Ausgestaltung der EU als Verhandlungs- und Konkordanzsystem
Zur Förderung einer europäischen Identitätsbildung kann insbesondere auch eine Demokratisierung politischer Entscheidungsverfahren und eine Ausweitung von Partizipationsmöglichkeiten auf europäischer Ebene beitragen. ${ }^{31}$ Angesichts der unzureichenden unmittelbaren demokratischen Legitimation der EU32 besteht grundsätzlich ein großer Handlungsbedarf. Das institutionelle Demokratiedefizit33 bestimmt seit dem Vertrag von Maastricht die politische wie wissenschaftliche Debatte um die Reform der Europäischen Union. Auch die Bürger erachten die Union als nicht ausreichend legitimiert, weil sie - zu Recht - auch ein demokratisches System auf EU-Ebene erwarten. Daher können sie sich auch kaum mit ihr identifizieren. Die heutige Europaskepsis vieler Unionsbürger beruht denn auch vor allem auf der Einschätzung, dass „ihre Stimme in der Europäischen Union nicht zählt". Immerhin 59 Prozent der EU-Bürger sind dieser Ansicht. ${ }^{34}$ Obwohl die Bevölkerung die Abgeordneten des Europäischen Parlaments alle fünf Jahre wählt, bietet Brüssel aus ihrer Sicht zu wenige Partizipationsmöglichkeiten, da wichtige Vorhaben wie die Einführung des Euro oder die Osterweiterung im Jahre 2004 ohne eine unmittelbare Mitsprache erfolgt sind. Die Bürger fühlen sich als bloßes Objekt europäischer Entscheidungen, die sie nicht beeinflussen können und nachträglich - beispielsweise auch in ex-post-Referenden wie denen zum Europäischen Verfassungsvertrag - „abzunicken“ haben.

Seit Beginn der 90er Jahre wurden immer wieder Forderungen laut, die EU müsse bürgernäher werden und den Menschen mehr Möglichkeiten der Beteiligung an der europäischen Politik einräumen. Bis heute ist dies jedoch trotz mehrerer Reformen des Primärrechts nicht in ausreichendem Maße gelungen. Auch der im Oktober 2004 unterzeichnete Verfassungsvertrag enthält zwar einige Neuerungen zur Verbesserung der demokratischen Legitimation der Europäischen Union, sein demokratischer Zugewinn ist allerdings zu Recht als „nicht sehr groß“ eingestuft worden ${ }^{35}$. Es besteht daher auch dann weiterhin Handlungsbedarf, wenn der Vertrag in seiner jetzigen Form tatsächlich in Kraft treten sollte, was derzeit allerdings ohnehin unwahrscheinlich ist.

Eng verknüpft mit dem institutionellen Demokratiedefizit ist das Öffentlichkeitsdefizit der EU,36 das für viele den Kern des europäischen Demokratiedefizits darstellt ${ }^{37}$. Auch wenn eine europäische Kommunikationsgemeinschaft als im Entstehen begriffen angesehen werden kann, ${ }^{38}$ so ist sie gleichwohl bislang nur schwach ausgeprägt. Nur die Herausbildung einer europäischen Öffentlichkeit vermag jedoch zu bewirken, dass sich tatsächlich bestehende und/oder konstruierte Gemeinsamkeiten zwischen den Bürgern auch in der subjektiven Wahrnehmung umsetzen können. ${ }^{39}$ So ist eine der Funktionen von Öffentlichkeit die Identitätsbildung der Gesellschaft, indem Bürger - über die Öffentlichkeit vermittelt - dauerhaft die Gesellschaft beobachten, an ihr teilhaben und sie als die ihre begreifen. ${ }^{40}$ Die Ausbildung einer europäischen Identität hängt daher entscheidend von der Entstehung einer europäischen Öffentlichkeit ab. ${ }^{41}$

Ein maßgeblicher Grund 42 für die nur schwach ausgeprägte europäische Öffentlichkeit ist die institutionelle Ausgestaltung der EU als Verhandlungs- und Konkordanzsystem. ${ }^{43}$ Das europäische Governance-System ist bei der Entscheidungsfindung seiner Organe strukturell auf Konsens angelegt. Als eine nicht- 


\section{$\mathrm{C} \cdot \mathrm{A} \cdot \mathrm{P}$}

majoritäre Demokratieform steht sie im Gegensatz zur Konkurrenzdemokratie, die Konflikte im Wesentlichen mit Hilfe des Mehrheitsprinzips bewältigt. ${ }^{44}$ So dominiert im Rat die Einstimmigkeitsregel. ${ }^{45}$ Im Europäischen Parlament ergibt sich ein Zwang zur Bildung einer großen Koalition, da bei den entscheidenden Abstimmungen im legislativen Bereich mehr als 50 Prozent der Stimmen erforderlich sind und keine der beiden großen Fraktionen diese Mehrheit alleine zu Stande bringt. Und schließlich werden dieVorschläge der Kommission - dem Kollegialitätsprinzip folgend - von der Kommission nach außen als Ganzes vertreten. Auch die europäischen Organe untereinander entwickeln nur in geringem Maße langfristige und für den Bürger deutlich erkennbare eigene - und damit auch unterschiedliche - Standpunkte, sind sie doch im Rahmen des „institutionellen Gleichgewichts“ zu einem von Kompromissen bestimmten Zusammenwirken bei der Gesetzgebung gezwungen. Der Charakter der Union als Verhandlungssystem führt außerdem zu einem weitgehenden Ausschluss der Öffentlichkeit bei der Rechtssetzung. Von den drei insoweit maßgeblichen Organen tagt nur das Europäische Parlament öffentlich. Hinzukommt das Selbstverständnis der EU-Institutionen und ihrer Mitglieder sowie vieler Experten. Diese begreifen die Europäische Union noch immer vor allem als technokratisches, apolitisches Regime zur effektiven Lösung europäischer Probleme, die frei wären von politischen Wertvorstellungen.

Die "geheime Kabinettsdiplomatie" im Rat sowie der notwendige Konsens zwischen den Organen führen dazu, dass bestehende politische Konflikte nicht offen und argumentativ ausgetragen und alternative politische Konzepte, Ansätze und Lösungsmöglichkeiten dem Publikum vorenthalten werden. ${ }^{46}$ Auch wird nicht erkennbar, dass die Entscheidungen, die getroffen werden, alle Bürger in der Europäischen Union betreffen. Damit verbirgt die europäische Politik ihren Entscheidungscharakter und ihre Entscheidungen erscheinen als alternativlos. Und mangels öffentlicher Kontroversen über die Politik der Union wird der Debatte die lebensnotwendige Nahrung vorenthalten. Die Dominanz der konsens- und verhandlungsorientierten Entscheidungsfindung in der EU führt daher zu einer systematischen Entpolitisierung europäischer Politik. Der institutionellen Bearbeitung von europäischen Themen wird gerade jene Konfliktsstruktur vorenthalten, die die öffentlichen Meinungsbildungsprozesse in Gang hält und die an Konfliktkonstellationen interessierten Medien bedienen könnten. ${ }^{47}$ Unter solchen Bedingungen findet die Europäische Union selbst nur ein begrenztes Maß an Aufmerksamkeit bei den Bürgern. Umgekehrt haben die politischen Akteure nur ein vermindertes Interesse daran, an der Herstellung öffentlicher Meinungen mitzuwirken. Dasselbe gilt für die Medien, fehlt es doch am Anreiz, über Europa im Allgemeinen und Europapolitik im Besonderen zu berichten, wenn kein hinreichend großes Publikum vorhanden ist. 48

Europäische politische Kommunikation zeichnet sich durch ein "Zuwenig an Streitkommunikation aus“49: Das europäische Öffentlichkeitsdefizit besteht „in einer mangelnden Dynamik europapolitischer Kommunikation und ihrer Schwerfälligkeit, die latent vorliegenden Streitthemen in eine Debatte zwischen den Europäern zu überführen. (...) Öffentlich [massenmedial] inszenierte Konflikte können insofern einen zentralen Beitrag zur Deckung des gegenseitigen Beobachtungsbedarfs eines funktional differenzierten und territorial segmentierten Mehrebenensystems des Regierens leisten (...). Eine polarisierte öffentliche Meinung verstärkt sogar die Dynamik von Öffentlichkeit, weil sie auf Seiten der herrschenden Zuordnungsprobleme aufwirft und damit Anlass gibt, öffentliche
Systematische Entpolitisierung europäischer Politik 


\section{$\mathrm{C} \cdot \mathrm{A} \cdot \mathrm{P}$}

Meinungskonflikte auszutragen. [Es kann deshalb erwartet werden,] dass erst durch die generelle Konfliktorientierung einer europäischen Öffentlichkeit und ihrer Akteure die europäischen politischen Zusammenhänge für eine massenmediale Präsentation zugeschnitten werden, um damit den vielfach beklagten Defiziten einer medialen Infrastruktur zur Vermittlung europäischer Politik auf wirksame Weise entgegenzutreten." ${ }^{50}$ Eine stärkere Politisierung europäischer Politik ist folglich Voraussetzung für die Herausbildung einer europäischen Öffentlichkeit. 51

\section{Möglichkeiten europäischer identitätsstiftender Politik}

\subsection{Eindämmung von Systemdynamik und -komplexität}

Wie ausgeführt, erschwert die Andersartigkeit der EU gegenüber Nationalstaaten „Maastricht als Dauerzustand“ und herkömmlichen Staatenverbindungen die Identifikation. Dem Handlungsspielraum beim Abbau der damit verbundenen Systemdynamik und -komplexität sind jedoch enge Grenzen gesetzt. Die Dynamik des politischen Systems wird auch in Zukunft ein konstitutives Merkmal der EU bleiben. Auch der Vertrag über eine Verfassung von Europa bedeutet wie alle vorangegangenen Verträge nur eine weitere Wegmarke und wird - sofern eine Ratifizierung durch alle Mitgliedstaaten überhaupt stattfinden wird - nicht die letzte Primärrechtsänderung in der Geschichte der europäischen Integration gewesen sein. Auch intendiert derVertrag keinen finalen Grundlagentext über die Ausgestaltung des politischen Systems der EU. Daneben sieht es nicht so aus, dass der fundamentale Dissens zwischen den Mitgliedstaaten über die Zielperspektive und damit über die Tiefe und Richtung des Integrationsprozesses in absehbarer Zeit überwunden werden könnte. Die endgültige Gestalt der EU wird daher unbestimmt bleiben. Zwar können die Referenden in Frankreich und den Niederlanden als Negativ-Votum gegen weitere Erweiterungen gewertet werden ${ }^{52}$ und bestehen auch in anderen EU-Staaten große Vorbehalte hinsichtlich der Aufnahme neuer Mitglieder, insbesondere bezüglich der Türkei. Die bereits beschlossenen Erweiterungen um Rumänien und Bulgarien werden aber langfristig nicht die letzten sein, sondern vom Einzelfall abhängen. Allerdings erscheint es angesichts der starken Vorbehalte der Bürger ratsam, - zumindest vorerst - keine neuen Beitrittsperspektiven (über Kroatien, die Türkei und die Balkan-Staaten hinaus) zu eröffnen und attraktive Alternativkonzepte jenseits einer EU-Vollmitgliedschaft zu entwickeln. Eine abschließende Definition der EU-Grenzen ist aber weder politisch durchsetzbar noch sinnvoll im Hinblick auf die Handlungs- und Gestaltungsfähigkeit der Union.

Anerkennung des „Baustellencharakters“ der EU
Allerdings könnte der fehlende Bezugsrahmen vom Ansatz her dadurch abgemildert werden, in dem der "Baustellencharakter" der EU als positives Strukturmerkmal der EU anerkannt wird. Von der EU sollte mehr das Bild von einem flexiblen Gebilde, das gerade aufgrund seiner Systemdynamik über mehr Handlungsfähigkeit als die starren Nationalstaaten verfügt, vermittelt und damit ein vermeintliches Defizit positiv gewendet werden. In diesem Zusammenhang gilt es zudem, "Maastricht als Dauerzustand“ anzuerkennen, da zukünftig keine grundsätzlichen Änderungen an der politischen Ordnungsform der EU zu erwarten sind. ${ }^{53}$ Die Besonderheit der Union als Herrschaftsverband eigener Art wird sich nicht in einer Staatswerdung auflösen, sondern trotz Veränderungen im institutionellen Gefüge und in der Aufgabenstellung fortbestehen. Insbesondere bleiben die Mitgliedstaaten die "Herren der Verträge". Folglich sollte von der Vorstellung Abschied genommen werden, zur Erhaltung des dynamischen Prozesses sei es 


\section{$\mathrm{C} \cdot \mathrm{A} \cdot \mathrm{P}$}

erforderlich, immer neue Gemeinschaftsaufgaben zu formulieren. Stattdessen darf es Kompetenzen der EU nur geben, wenn ein konkreter europäischer Mehrwert erkennbar ist, d.h. dass konkrete Aufgaben besser und effektiver auf europäischer als auf nationaler Ebene erfüllt werden können. Das gilt auch als Maßgabe für die Überarbeitung des Finanzrahmens ab dem Jahr 2014.

Aber auch an der grundsätzlichen Systemkomplexität der EU lässt sich nur schwerlich etwas ändern. Die Union wird allein schon auf Grund ihrer Größe ein komplexes Verhandlungssystem mit einer Vielzahl von staatlichen und privaten Institutionen sowie Netzwerken, die an der Entscheidungsfindung beteiligt sind, bleiben. Darin wird weiterhin das Konsensprinzip vorherrschen, die EU also auch weiterhin als Konkordanzsystem zu charakterisieren sein. Die Dominanz des Einstimmigkeitsprinzips kann als Ausdruck der Notwendigkeit angesehen werden, angesichts eines hochgradig fragmentierten Gemeinwesens ein hohes Maß an Machtteilung und Autonomie der einzelnen Mitgliedstaaten und deren nationalen Gesellschaften zu gewährleisten. Als eine Vereinigung heterogener Gesellschaften, die noch keine gemeinsame Identität als politische Gemeinschaft herausgebildet hat, ist die EU auch zukünftig auf die integrierenden Effekte einer prinzipiell konsensualen Entscheidungsfindung angewiesen. ${ }^{54}$ Da es noch an den Voraussetzungen für eine umfassende Anwendung des Mehrheitsprinzips fehlt, sind einer Ausweitung enge Grenzen gesetzt. ${ }^{55}$ Auch weiterhin werden daher die meisten Entscheidungen auf Grund der notwendigen Einstimmigkeit eine Kompromisslösung erfordern, welche die verantwortlichen Akteure nur schwerlich erkennen lässt.

Die Systemkomplexität könnte aber durch eine klarere Abgrenzung und Zuordnung der Kompetenzen zwischen EU und Mitgliedstaaten zumindest etwas entschärft werden. Dann wäre es für den Bürger erkennbarer, welche Ebene für welche Entscheidungen zuständig ist und wen er daher für Erfolge wie Misserfolge politisch verantwortlich machen kann. Erforderlich ist dafür eine transparente und verständliche Kompetenzverteilung. Dies gestaltet sich allerdings von vornherein schwierig angesichts der Tatsache, dass die Kompetenzverteilung der EU seit Beginn nicht dem Trennprinzip, sondern dem Politikverflechtungs-Modell folgt, so dass die meisten Kompetenzen nicht getrennt sind, sondern sich überlagern. ${ }^{56} \mathrm{Zu}$ denken wäre an eine bipolare Kompetenzordnung mit Primär- und Partialkompetenzen. ${ }^{57}$ Eine strikte bipolare Kompetenzordnung, nämlich eine klare Trennung der Kompetenzbereiche in dem Sinne, dass keine konkurrierenden bzw. gemeinsame Zuständigkeiten von EU und den Mitgliedstaaten bestehen, und eine Entkoppelung der politischen Willensbildung beider Ebenen, d.h. jede Ebene hat eine eigene Verwaltung und Gerichtsbarkeit, ist hingegen keine realistische Option, weil praktisch nicht umsetzbar und auch politisch kaum durchsetzbar. Zudem ist eine völlige Trennung der derzeit überwiegend geteilten Kompetenzen aufgrund der Interdependenzen öffentlicher Aufgaben und Problemlagen als nicht effektiv und problemlösungsorientiert anzusehen. ${ }^{58}$ Außerdem können der EU bestimmt Kompetenzen ausdrücklich vorenthalten werden, in dem positive Kompetenzzuweisungsnormen an die Union um negative Kompetenzbestimmungen ergänzt werden, welche faktisch wie ausschließliche Zuständigkeiten der Mitgliedstaaten wirken würden.59 Daneben könnte die Aufstellung eines übersichtlichen Kompetenzkatalogs, in dem die Tätigkeitsfelder der EU aufgeführt und um die Bereiche zu ergänzen sind, in denen die Union keine Zuständigkeit hat, die Arbeitsteilung zwischen der EU und den Mitgliedstaaten sichtbar machen. Die Auflistung der Kompetenzen würde die eigentlichen (funktional ausgerichteten) 


\section{$\mathrm{C} \cdot \mathrm{A} \cdot \mathrm{P}$}

Kompetenznormen, die präziser und enger zu fassen sind, um weitere Zentralisierungseffekte zu vermeiden, jedoch nicht ersetzen, sondern auf diese verweisen. Eine Umstellung des derzeitig aufgabenorientierten Kompetenzsystems auf allein sachgebietsbezogene Zuständigkeitsabgrenzungen in einem Kompetenzkatalog ohne konkrete Einzelermächtigungen ist abzulehnen, weil reine Kompetenzkataloge im Gegensatz zu differenzierten Kompetenzregelungen eine extensive Kompetenzausübung stärker begünstigen. Dabei wäre eine Definition und Systematisierung der Kompetenzen nach klaren Kategorien und anschließend eine eindeutige Zuordnung der einzelnen Befugnisnormen zu einer bestimmten Kompetenzkategorie vorzunehmen.

\subsection{Abschwächung der Geltung des nationalstaatlichen Prinzips}

Strategien zur Abschwächung der Geltung des nationalstaatlichen Prinzips müssten einerseits bei der Kompetenzverteilung zwischen den Mitgliedstaaten und der EU ansetzen und andererseits eine neue Begründungslogik für den europäischen Einigungsprozess anbieten. Wie bereits angesprochen, geht es dabei nicht darum, die Identifikation mit dem Nationalstaat aufzulösen, sondern das Zugehörigkeitsgefühl zur EU zu stärken. Nationale und europäische Identität schließen sich nicht aus, sondern können - wie andere Identitäten auch - nebeneinander bestehen. Die europäische Identität würde nicht zwingend auf Kosten der nationalen Identität wachsen, sondern es würde eine Identitätserweiterung stattfinden. ${ }^{60}$ Dies liegt darin begründet, dass Identität kein Nullsummenspiel ist. Jeder Mensch bildet als Mitglied des politischen Gemeinwesens mehrere Identitäten aus, so dass multiple soziale Identitäten möglich sind. ${ }^{61}$ So wie lokale, regionale und nationale Bindungen bestehen, kann das Identitätsbewusstsein um eine weitere - die europäische Dimension - ergänzt werden, ohne dass die anderen Bezüge dadurch verschwinden.

Potenziellen Strategien, die das nationale Prinzip abmildern, sind allerdings von vornherein erhebliche Grenzen gesetzt. Denn auch wenn angesichts stetig wachsender globaler Herausforderungen zukünftig von einer immer engeren Kooperation der Mitgliedstaaten im Bereich der Außenpolitik und der inneren Sicherheit auszugehen ist, erscheint eine Vergemeinschaftung dieser "harten“ Souveränitätsbereiche in der nahen Zukunft als nicht realistisch. ${ }^{62}$ Auch werden die Mitgliedstaaten auf absehbare Zeit ihre Kompetenzen im Bereich der Bildungspolitik behalten. Angesetzt werden kann daher nicht auf der europäischen, sondern allenfalls der nationalen Ebene. Insbesondere der Bereich der nationalen Schul- und Bildungspolitik steht hier im Mittelpunkt der Diskussion. ${ }^{63}$ Diese muss sicherstellen, dass alle ihre Bürger die Kenntnisse und Fähigkeiten haben, vollumfänglich am demokratischen Leben der EU auf nationaler und europäischer Ebene teilzunehmen. Andernfalls laufen die bestehenden Partizipationsmöglichkeiten weitgehend ins Leere. Ziel muss es insbesondere sein, das immense Wissensdefizit der Bürger über die EU abzubauen und die Relevanz der auf europäischer Ebene getroffenen Entscheidungen für den einzelnen Bürger zu vermitteln. Nur so lässt sich auch den vielfach verbreiteten Fehlvorstellungen und Vorurteilen über die Union die Grundlage entziehen. Erst die Kenntnis vom Entstehungsprozess der Entscheidungen europäischer Politik ermöglicht auch die Erkennbarkeit der Einwirkungsmöglichkeiten, die den Bürgern und ihren Vertretern jeweils zur Verfügung stehen. Letztendlich geht es aber auch darum, die EU als fester und dauerhafter Bestandteil des politischen Systems des eigenen Nationalstaates und 


\section{$\mathrm{C} \cdot \mathrm{A} \cdot \mathrm{P}$}

dessen nationaler Gesellschaft und Öffentlichkeit zu begreifen, und nicht als etwas "Fremdes", das außerhalb des jeweiligen Mitgliedstaates liegt. Um diese Ziele zu erreichen, spielt die Vermittlung ausreichender Kenntnisse über das politische System der EU, über gemeinsame europäische Wertgrundlagen sowie kultureller Unterschiede ebenso eine entscheidende Rolle wie die Vermittlung des Verständnisses von den politischen und wirtschaftlichen Interdependenzen und Problemlagen, denen sich jeder europäische Staat im Verhältnis zu den anderen EUStaaten und auch anderen Staaten und Regionen außerhalb Europas ausgesetzt sieht. Die Lehrpläne müssen so ausgerichtet sein, dass auch jeweils die europäische Dimension in den Bereichen Politik, Geschichte, Geographie, Religion und Sprachen herausgestellt wird. Gefordert ist daher eine Europäisierung der Bildungsinhalte, 64 insbesondere die "Einübung eines gemeinsamen europäischen Geschichtsbildes (...), [das] den Kampf um die Vergangenheit der kriegerischen europäischen Geschichte methodisch auflöst - dies allerdings gerade nicht, indem europäische Geschichte neben der oder zusätzlich zur jeweiligen Nationalgeschichte dargestellt, sondern indem sie in nationale und regionale Identitäten und Geschichten integriert wird"65. Außerdem gilt es, die Erlernung anderer europäischer Sprachen, insbesondere von Englisch als europäische Verkehrssprache, noch stärker als bisher zu forcieren, um die Kommunikationsfähigkeit der Bürger zu stärken und damit auch die europäischen Öffentlichkeit zu befördern. Freilich werden alle diese Maßnahmen, selbst wenn man sie sofort umsetzen würde, frühestens in vielen Jahren Wirkung zeigen können.

Zusätzlich benötigt die EU einen neuen - oder genauer ergänzenden - Begründungszusammenhang, der den elementaren Bedarf an Orientierung abdeckt. Die EU als Friedensprojekt bleibt auch zukünftig das zentrale Motiv für die europäische Einigung. Die alten Begründungen sind aber nicht mehr ausreichend und müssen daher ergänzt werden, um den Bürgern den konkreten Mehrwert des Integrationsprojekts zu vermitteln. Heute erscheint es für die Bürger, aber auch für viele Eliten unklar, wofür die EU überhaupt gebraucht wird. Das Projekt Europa gilt vielen Bürgern nicht als Antwort auf die vielseitigen Herausforderungen einer sich globalisierenden Welt, sondern vielmehr als Teil des Problems. Angesichts von grenzüberschreitenden Problemlagen und Bedrohungen für die Sicherheit aller europäischer Staaten, bei denen nationale Alleingänge keine wirkungsvolle Lösung versprechen, Sachproblemen, die ihrem Wesen nach nur geo-regional gelöst werden können oder bei denen der Aufwand der Problemlösung für einen einzelnen Staat unverhältnismäßig hoch ist, und angesichts wirtschaftlicher Inderdependenzen und eines weltweiten Wettbewerbs, gepaart mit einem Verlust an politischer Steuerungsfähigkeit aufgrund weltweit agierender ökonomischer Akteure sowie einer schwindenden geopolitischen Bedeutung Europas seit Beginn der 90er Jahre bietet eine verstärkte Nationalisierung und Abschottung nach außen, wie sie von vielen gefordert und zum Teil auch verstärkt praktiziert wird, allerdings keine wirkungsvolle Strategie, um die Folgen der Globalisierung adäquat auszugleichen. „Die Antwort auf [die Frage nach einer neuen Begründungslogik] liegt in den neuen Konstellationen und Bedingungen der Weltpolitik: Es geht um die künftige Gestaltungskraft Europas in einer neuen globalen Ordnung. Nach dem Ende des Kalten Krieges, mit dem Aufkommen neuer wirtschaftlicher und politischer Mächte in Asien und Südamerika und durch die Globalisierung von Ökonomie und Sicherheit wird die Zukunft Europas zunehmend durch Entwicklungen außerhalb des alten Kontinents entschieden. Es droht erneut die Gefahr einer schleichenden Marginalisierung des europäischen Kontinents.

Entwicklung einer neuen Begründungslogik 


\section{$\mathrm{C} \cdot \mathrm{A} \cdot \mathrm{P}$}

Europa muss nicht nur auf diese Entwicklungen reagieren, es hat vielmehr das Potenzial, die Regeln der neuen ökonomischen und politischen Weltordnung nach seinen Vorstellungen mitzugestalten." 66 Die europäische Einigung als wirtschaftliches, politisches und sicherheitspolitisches Projekt in einer sich rasant verändernden Welt, die das Potenzial hat, diese nach den eigenen Vorstellungen mitzugestalten - dies gilt es, den Bürgern zu vermitteln. In diesem Zusammenhang dürfte ein neues europäisches Großprojekt hilfreich sein, das die Notwendigkeit der Existenz der Union zu unterstreichen vermag und ihren Mehrwert für den Bürger konkret deutlich macht. Zu denken wäre hier an den Themenkomplex Sicherheit, der die äußere wie innere Sicherheit umfasst und zivile wie militärische Aspekte beinhaltet. Insbesondere in der Außen- und Sicherheitspolitik ist die Erwartungshaltung der EU-Bürger an die EU hoch, was entsprechende Umfragen von Eurobarometer zeigen. ${ }^{67}$ Auch ist eine engere sicherheitspolitische Kooperation für die Mitgliedstaaten und ihre Bürger mit Vorteilen verbunden, welche die Nationalstaaten alleine nicht zu erzielen vermögen. Der europäische Mehrwert liegt nahezu auf der Hand, was sich auch daran zeigt, dass in den letzten Jahren gerade im Bereich der inneren und äußeren Sicherheit eine engere Kooperation der EU-Staaten stattgefunden hat. Diese besteht allerdings bislang noch weitgehend aus Einzelmaßnahmen, die ohne überwölbendes Konzept lose nebeneinander stehen. Der Themenkomplex Wirtschaft und Soziales erscheint dagegen abgesehen von Einzelmaßnahmen - als Großprojekt nicht geeignet, auch wenn was Eurobarometer-Umfragen zeigen - die EU die Erwartungen der Bürger in der Wirtschafts- und Sozialpolitik nicht erfüllt und das Votum der Franzosen im Referendum über den europäischen Verfassungsvertrag als "Nein" gegen das angeblich neoliberale Wirtschaftsmodell der EU gewertet werden kann. Dies deswegen, weil aufgrund der Heterogenität der nationalen Wirtschafts- und Sozialmodelle, denen zum Teil völlig andere normative Wertungen hinsichtlich der Sozialstaatlichkeit zu Grunde liegen, eine europäisches Wirtschafts- und Sozialunion unrealistisch ist. Es ist nicht davon auszugehen, dass die Mitgliedstaaten wichtige Teile des letzten Horts ihrer nationalen Souveränität, der Sozialpolitik, an Brüssel abgeben werden. Zudem ist der europäische Mehrwert einer einheitlichen europäischen Sozialpolitik höchst fraglich. ${ }^{68}$

Auch insoweit ist allerdings zu konstatieren, dass die Vermittlung eines neuen Narrativs ein schwieriges und langfristig angelegtes Unterfangen sein dürfte. Im Gegensatz zu den Zeiten des Ost-West-Konflikts sind die aktuellen Herausforderungen und Bedrohungen nur diffus und oftmals nicht unmittelbar erkenn- und erfahrbar. Gleichwohl ist das Potenzial der Legitimation der EU durch ihr Verständnis als Gestaltungsmacht in einer globalen Perspektive bislang bei weitem nicht ausgeschöpft worden.

\subsection{Mehr Partizipation}

In der seit Jahren andauernden Debatte um eine Behebung oder wenigstens Redu-

Präsidentieller Demokratisierungsansatz zierung des Demokratiedefizits der EU sind eine Vielzahl an Reformvorschlägen auf der Basis unterschiedlicher Demokratisierungskonzepte gemacht worden. ${ }^{69}$ Eine Ausweitung der Partizipationsmöglichkeiten der Bürger am politischen Entscheidungsprozess in der EU versprechen sich viele von einer Aufwertung des Europäischen Parlaments, insbesondere durch die Einräumung des Rechts zur Bestellung der Kommission. ${ }^{70}$ Anstelle einer solchen Parlamentarisierung der Union sollte die derzeitige duale Legitimation der Europäischen Union allerdings 


\section{$\mathrm{C} \cdot \mathrm{A} \cdot \mathrm{P}$}

um Strategien ergänzt werden, die sich nicht am Modell einer rein repräsentativen, parlamentarischen Mehrheitsdemokratie orientieren, wie insbesondere der präsidentielle Demokratisierungsansatz. ${ }^{71}$ Dieser plädiert dafür, den Kommissionspräsidenten im Wege einer Direktwahl durch die Unionsbürger zu bestimmen. ${ }^{72}$ Daneben wären auch europaweite Referenden ${ }^{73}$ bei Änderungen des europäischen Primärrechts und Beitritten denkbar. Auch wenn es gelingen würde, ein Referendum in jedem Land der EU am selben Tag abzuhalten, um zu verdeutlichen, dass Gegenstand der Abstimmung europäische und nicht nationale Politik ist, blieben es jedoch gleichwohl nationale Abstimmungen von rein konsultativen Charakter, da andernfalls die Staatlichkeit der Mitgliedstaaten angetastet werden würde, weil die Mitgliedstaaten nicht mehr „Herren der Verträge“ wären. Aufgrund der Komplexität von Änderungsverträgen und mangels Alternativangebot bei einer entsprechenden Abstimmung wäre es ohnehin vorzuziehen, nach SchweizerVorbild Referenden allenfalls zu einzelnen konkreten Maßnahmen oder Projekten der EU durchzuführen. Außerdem könnte die europäische Bürgerinitiative, die im europäischen Verfassungsvertrag in Artikel I-47 Absatz 4 vorgesehen ist, ${ }^{74}$ auch ohne dessen Ratifizierung umgesetzt werden.

Während die vorgenannten Vorschläge darauf abzielen, das Demokratiedefizit durch eine Ausweitung der unmittelbaren Einflussmöglichkeiten der europäischen Bürger auf den Politikprozess der europäischen Institutionen zu beheben, sehen andere in der nationalen die maßgebliche Ebene, wo Reformen anzusetzen haben. Insbesondere eine Stärkung der Rolle der nationalen Parlamente im europäischen Entscheidungsprozess wird gefordert. ${ }^{75}$ Im Verfassungsvertrag ist insoweit die Etablierung eines Frühwarnmechanismus vorgesehen, der den nationalen Parlamenten die Möglichkeit bietet, einen Einspruch gegen Vorschläge der Kommission zu erheben, wenn sie das Subsidiaritätsprinzip verletzt sehen. Die entsprechenden Regelungen könnten auch ohne eine Änderung des Primärrechts und daher unabhängig von einer Ratifizierung des Verfassungsvertrags in Kraft treten. Dadurch würden die nationalen Parlamente bei der Kontrolle der nationalen Regierungen gestärkt und ihre direkte Beteiligung am EU-Gesetzgebungsprozess ausgeweitet. ${ }^{76}$ Außerdem wäre auch eine bessere "Verzahnung" der nationalen und europäischen Ebene zu empfehlen.

\subsection{Politisierung europäischer Politik}

\subsubsection{Institutionelle Reformen}

Wie oben ausgeführt ist der Mangel an Politik institutionell-strukturell bedingt. Man wird daher eine stärkere Politisierung auch nur auf diesem Wege erreichen können. Nur wenn sich die entsprechende "institutionelle Anreizstruktur“ ändert, wird sich auch das Verhalten der Akteure - gezwungenermaßen - ändern und sich die europäische Öffentlichkeit entscheidend weiterentwickeln. ${ }^{77}$ Es bedarf folglich institutioneller Reformen, und zwar solcher, welche eine "Streitkommunikation" befördern und darüber hinaus die Entscheidungsträger unmittelbar an die betroffenen Bürger koppeln. ${ }^{78}$

Änderungen der institutionellen Ordnung können dann relevante Impulse für die Herausbildung einer europäischen Öffentlichkeit liefern, wenn die auf der europäischen Ebene handelnden Akteure stärker als bisher dazu gezwungen werden, für ihre Positionen in der Öffentlichkeit Unterstützung zu erlangen, und das europäi-

Änderung der „institutionellen Anreizstruktur" 


\section{$\mathrm{C} \cdot \mathrm{A} \cdot \mathrm{P}$}

Direktwahl des Kommissionspräsidenten und Europäisierung des Wahlsystems zum EP

Folgewirkung: "Streikommunikation“ sche Publikum stärker als bisher dazu bewegt wird, sich für die europäische Politik zu interessieren. ${ }^{79}$ Denn Öffentlichkeit wird für Entscheidungsträger erst dann bedeutsam, wenn sie über eine Sanktionsmacht verfügt, die politischen Akteure also von der Meinung des Publikums abhängig sind. ${ }^{80}$ Ist dies der Fall, werden sie um die Bürger in der Öffentlichkeit werben müssen. Für die Bürger gilt umgekehrt, dass sie sich erst dann mehr für die über Öffentlichkeit vermittelte Politik interessieren, wenn die relevanten Institutionen für sie maßgebliche Entscheidungen treffen und sie auf deren Politik auch Einfluss nehmen können. Ein solcher Zusammenhang ist in Demokratien insbesondere dadurch institutionalisiert, dass die Besetzung politischer Ämter durch Wahlen erfolgt. Gleichzeitig kann damit auch die stärkere Ausbildung eines europäischen Kommunikationsraums befördert werden, in dem politische Argumente ausgetragen und Debatten über zu treffende europäische Entscheidungen geführt werden, und über den die Bürger mittelbar am europäischen Entscheidungsprozess beteiligt werden und diesen kontrollieren können.

Entsprechend dem präsidentiellen Demokratisierungsansatz sollten die Partizipationsmöglichkeiten der Bürger aber nicht dadurch ausgebaut werden, dass das Europäische Parlament aufgewertet wird und das Recht zur Wahl des Kommissionspräsidenten erhält, sondern dieser sollte direkt von den Bürgern gewählt werden. ${ }^{81}$ Außerdem sollte das Wahlsystem zum Europäischen Parlament europäisiert werden. ${ }^{82} \mathrm{Zu}$ denken ist insbesondere an die Einführung transnationaler Wahllisten, wobei beispielsweise zehn Prozent der Sitze nicht national vergeben werden, sondern europaweit über einheitliche europäische Parteilisten. Die Zuteilung der übrigen Mandate an die einzelnen Mitgliedstaaten erfolgt wie bisher aufgrund einer nationalen Kontingentierung.

Beide Vorschläge würden eine Reihe miteinander zusammenhängender Folgewirkungen haben: Neben einem unmittelbaren Einfluss auf die Bestellung der Exekutive bewirkt die Direktwahl des Kommissionspräsidenten eine stärkere Personalisierung europäischer Politik, mit der bislang kaum ein konkretes Gesicht verbunden wird. Indem er einen substanziellen Teil des europäischen Gemeinwesens repräsentiert, könnte der Kommissionspräsident verkörpern, was die politische Einheit der EU in der Praxis ausmacht. Die Aufstellung von Spitzenkandidaten für das Amt des Kommissionspräsidenten sowie transnationaler Wahllisten durch die europäischen Parteibündnisse bieten die Möglichkeit der Etablierung eines europäischen Parteiwesens als Bestandteil einer europäischen Öffentlichkeit und Zivilgesellschaft. Die europäischen Parteien in ihren Funktionen als Mittler zwischen Politik und Öffentlichkeit (Artikulations- und Vermittlungsfunktion im Prozess der Willensbildung und Politikentscheidung) sowie in ihrer Rekrutierungsfunktion (Zurverfügungstellung von politischem Personal) auf europäischer Ebene könnten gestärkt werden. ${ }^{83}$ Der Wahlkampf wäre transnational ausgerichtet, denn die Politiker der einzelnen Parteien müssten sich gleichermaßen in allem Mitgliedstaaten den Wählern stellen und nicht nur in ihren jeweiligen Heimatländern. Die Wahlen zum Europäischen Parlament würden damit ihren rein nationalen Charakter verlieren. Anstelle der bislang - jedenfalls aus der Sicht der Bürger - vorherrschenden „Propaganda" einer alternativlosen Politik durch Technokraten würde die Profilierung unterschiedlicher politischer Konzepte und Lösungsansätze für europäische Problemlagen durch Politiker und Parteien treten. Streit, Polarisierung und kontroverse Debatten als Wesensmerkmale einer lebendigen demokratischen Öffentlichkeit würden die Aufmerksamkeit und das Interesse an 


\section{$\mathrm{C} \cdot \mathrm{A} \cdot \mathrm{P}$}

europäischer Politik bei den Bürgern erhöhen, aber auch bei den politischen Akteuren und den Medien. Durch die auf Grund von institutionellen Reformen ausgelöste "Streitkommunikation" wäre das elementare Interesse aller drei Akteursgruppen am gemeinsamen Interaktionszusammenhang gegeben.

Dagegen ist durch eine - vielfach geforderte - Öffentlichkeit von Ratssitzungen das Transparenzdefizit der Legislativ- und Administrativverfahren der EU nur bedingt behebbar. Die Staats- und Regierungschefs der EU haben auf ihrem Gipfel im Juni 2006 beschlossen, dass insbesondere sämtliche Sitzungen der Ministerräte im Mitentscheidungsverfahren inklusive der Erklärungen der Ratsmitglieder zur Stimmabgabe zukünftig öffentlich sein werden, wie dies auch in Artikel I-24 Absatz 6 des Verfassungsvertrags vorgesehen ist. Es ist jedoch fraglich, ob sich dies in der Praxis bewähren wird. Es ist zu befürchten, dass die Ratstagungen zu bloßen Showveranstaltungen degenerieren und die wirklichen Entscheidungen in informellen Gesprächen außerhalb der offiziellen Ratssitzungen getroffen werden. Bei der Forderungen nach öffentlichen Ratssitzungen ${ }^{84}$ wird übersehen, dass der Ausschluss der Öffentlichkeit in Verhandlungssystemen wie der EU eine der Grundvoraussetzungen ist, um überhaupt zu Verhandlungslösungen zu kommen. ${ }^{85}$ Das Aushandeln inklusive der bekannten Tauschgeschäfte und Paketlösungen funktioniert grundsätzlich besser ohne Öffentlichkeit, da diese die agierenden Vertreter faktisch zur Verhärtung ihrer Standpunkte zwänge und damit Problemlösungen durch Kompromisse erschwert werden würden. Eine Umstellung auf öffentliche Sitzungen des Rates würde dessen Handlungsfähigkeit deshalb erheblich schwächen. Die Auflösung des Spannungsverhältnisses zwischen Öffentlichkeit und Effizienz könnte nur durch ein Abgehen vom Verhandlungsmodus erfolgen, indem gleichzeitig die prinzipielle Anwendung des Mehrheitsprinzips im Ministerrat eingeführt wird. Damit ist in den nächsten Jahren aber nicht zu rechnen.

\subsubsection{Mehr Dialog und Debatte}

Zur Herausbildung einer "Streitkommunikation" ist aber auch etwas erforderlich, was mit "mehr Dialog und Debatte“ bezeichnet werden kann. Entgegen der Vorstellung vieler Eurokraten, insbesondere der EU-Kommission, ist politische Öffentlichkeit in erster Linie keine "Angelegenheit besserer Information über die Organisation des politischen Europas und die Zuständigkeit seiner Institutionen, sondern die Wortmeldung der europäischen Bürger zu den Angelegenheiten, die sie nach dem eigenen Urteil unbedingt angehen"86. In dem aktuellen Weisbuch der Europäischen Kommission über eine europäische Kommunikationspolitik vom Februar 2006 erkennt diese nun auch ausdrücklich an, dass in der Vergangenheit mehr ein Monolog hin zum Bürger stattgefunden hat anstelle des notwendigen Dialogs mit dem Bürger. 87 Eine neue Kommunikationsstrategie aller europäischen Institutionen sollte dabei vor allem beinhalten, den notwendigen Austausch über „Für" und "Wider" eines neuen europäischen Projekts oder einer Einzelmaßnahme bereits während des EU-Entscheidungsverfahrens auf nationaler und europäischer Ebene zu organisieren. Ein positives Beispiel stellt hier die sehr umstrittene Dienstleistungsrichtlinie dar, wo eine breite, sogar europaweite Debatte schon im Stadium der Entscheidungsfindung zwischen den europäischen Institutionen, d.h. bevor der Ministerrat über eine Sache entscheidet, stattgefunden hat, und nicht erst im Zeitpunkt der nationalen Umsetzung, wenn an den auf EU-Ebene beschlossenen Verordnungen oder Richtlinien nichts mehr zu ändern

Öffentlichkeit von Ratssitzungen 


\section{$\mathrm{C} \cdot \mathrm{A} \cdot \mathrm{P}$}

Neue Wege zum Dialog mit den Bürgern ist. Zudem gilt es, bei den Bürgern kontinuierlich um Zustimmung für die europäische Politik zu werben, und zwar durch detaillierte Begründung jedes einzelnen Projekts in einem öffentlichen Interaktionsprozess. Gerade dies ist bei der Osterweiterung im Jahr 2004 versäumt worden, über die unter Ausschluss der breiten Öffentlichkeit entschieden worden ist. Die europäischen Institutionen haben darauf vertraut, dass die Bürger diesem Projekt schon ihre faktische Zustimmung erteilen werden. Stattdessen sieht sich die EU mit einer weit verbreiteten Ablehnungshaltung konfrontiert, die von diffusen Ängsten dominiert wird. Zur Behebung des „Kommunikationsdefizits" 88 der EU müssten auch die jeweiligen Alternativen zu einzelnen Vorhaben der EU dargelegt sowie die politischen und wirtschaftlichen Kosten und Nutzen einzelner Optionen aufgezeigt werden. Nur so kann dem derzeitigen Eindruck entgegengewirkt werden, europäische Politik sei alternativlos und jede Debatte sei eigentlich zwecklos, da das Ergebnis ohnehin schon feststehe. Ziel muss es daher sein, ein differenziertes Bild von Europa zu vermitteln, das unterschiedliche politische Optionen und kritische Aspekte zulässt.

Außerdem sollten neue Wege beschritten werden, um den Dialog mit den Bürgern $\mathrm{zu}$ generieren und zu organisieren. ${ }^{89}$ Zentral sind die Diskussion wichtiger EUInitiativen in den nationalen Parlamenten und die Weiterleitung der Ergebnisse der nationalen Debatten an die EU-Gremien vor der entsprechenden Entscheidung des Rates. Nationale Parlamente sind als unmittelbar legitimierte Repräsentanten der Völker der EU am besten geeignet, eine Debatte unter einer breiteren Öffentlichkeit anzustoßen und das Medieninteresse auf ein spezielles Thema zu lenken. Damit könnte auch stärker als bisher verhindert werden, dass nationale Akteure die europäische Gesetzgebung als „illegitimen Akt" der Brüsseler Bürokratie darstellen können. Auch eine verstärkte Einbeziehung europäischer Akteure in nationale Debatten wäre von Vorteil, um den Bürgern einen besseren Einblick in die Arbeit der europäischen Institutionen und deren Kompetenzen zu vermitteln. $\mathrm{Zu}$ denken ist insbesondere an regelmäßige Besuche von Abgeordneten des Europäischen Parlaments und Kommissaren in den Mitgliedstaaten, vor allem in den nationalen Parlamenten. Es sollten darüber hinaus nationale Bürgerforen organisiert werden, in denen öffentliche Debatten zu europäischen Fragestellungen unter Beteiligung von Politikern und Vertretern der Zivilgesellschaft stattfinden. Als Modell kann hier das "National Forum on Europe" in Irland dienen, das nach dem Scheitern des ersten irischen Referendums über den Vertrag von Nizza im Jahr 2001 gegründet worden ist. Das Forum versteht sich nicht als Förderer bestimmter Positionen wie beispielsweise der irischen Regierung oder der EU-Institutionen. Vielmehr soll in den Foren ein offener, unzensierter Dialog geführt werden. Außerdem ist an eine regelmäßigere Vermittlung politischer Prioritäten und Standpunkte der nationalen Regierungen in ihren nationalen Parlamenten, an ein jährliches Treffen nationaler und europäischer Parlamentarier zur Stärkung der öffentlichen Aufmerksamkeit für europäische Belange sowie die Herausgabe nationaler Informationsbulletins in regelmäßigen Abständen, die Informationen über die neuesten Vorschläge und Beschlüsse der EU enthalten und über die nationalen Medien möglichst weiträumig verbreitet werden, zu denken. 


\section{$\mathrm{C} \cdot \mathrm{A} \cdot \mathrm{P}$}

\section{Fazit}

Die Bilanz fällt damit unterschiedlich aus. An manchen Gründen für die nur schwach ausgeprägte europäische Identität wird sich nur schwerlich etwas ändern lassen, bei anderen besteht ein größerer Handlungsspielraum. Angesichts seiner fehlenden Stabilität und Dauerhaftigkeit sowie seiner Komplexität wird sich das politische System der EU auch in Zukunft als wenig identitätsstiftend erweisen. Allenfalls eine Anerkennung des "Baustellencharakters" der EU als positives Strukturmerkmal und eine transparentere Abgrenzung der Kompetenzen zwischen der EU und den Mitgliedstaaten versprechen hier etwas Abhilfe. Strategien zur Stärkung der europäischen Identität im Bereich der nationalen Schul- und Bildungspolitik sowie dieVermittlung einer neuen Begründungslogik für den europäischen Integrationsprozess werden erst langfristig Wirkungen zeigen.

Das größte Potenzial liegt im Bereich institutioneller Änderungen, mit denen eine stärkere Partizipation am europäischen Entscheidungsprozess verbunden ist und die eine Reduzierung des seit langem beklagten Demokratiedefizits beinhalten. Eine vermehrte Einbeziehung der Bürger kann zu einer stärkeren Auseinandersetzung mit dem Gemeinwesen anregen, somit die gegenseitige Anerkennung der Bürger als legitime Teilnehmer am kollektiven Willensbildungsprozess befördern und auch weiter gehende Identitätsbildungsprozesse in Gang setzen..$^{90}$ Der Ausbau der Partizipationsmöglichkeiten kann außerdem auch einer Ausweitung der Handlungsstrukturen politischer Öffentlichkeit dienen und $\mathrm{zu}$ einer Intensivierung der intermediären Vermittlungsstrukturen (insbesondere Medien, Parteien, Verbände) beitragen. Erst die verstärkte Ausbildung eines europäischen Kommunikationsraums, der den europäischen Bürgern die Wahrnehmung von Gemeinsamkeiten erlaubt, wird bei diesen ein belastbares Gemeinschaftsgefühl als Grundlage für eine stabile europäische Unterstützung des europäischen Projekts generieren.

Maßgeblich sind insoweit eine stärkere Politisierung europäischer Politik ${ }^{91}$ und der Aufbau einer europäischen "Streitkommunikation“. Dabei gilt es zu verdeutlichen: „Es gehört zu den kaum hinterfragten Grundüberzeugungen europäischer Eliten sei es in der Politik, sei es in der Wirtschaft -, dass kontroverse Debatten über Europa, die EU oder über einzelne europäische Politikthemen zu vermeiden sind, weil sie angeblich zur Herausbildung populistischer Strömungen und zur Gefährdung des europäischen Integrationsprozesses führen. Das Gegenteil ist der Fall! Streit und Politisierung sind konstitutiv für demokratische Meinungsbildung im öffentlichen Diskurs. Europa und die EU bilden hier keine Ausnahme. Eine stärkere Politisierung europäischer Themen würde die Aufmerksamkeit für Europa durch die nationalen Medien erhöhen und damit zu einer europäischen Öffentlichkeit und gleichzeitig zum Abbau des Demokratiedefizits der EU beitragen. Je mehr wir über nationale Grenzen hinweg über Fragen des europäischen Gemeinwesens als Europäer/innen streiten, desto mehr entsteht europäische Öffentlichkeit und eine kollektive europäische Identität." 92

Politisierung europäischer Politik

\section{Anerkennung des}

„Baustellencharakters“ der EU und eine transparentere Abgrenzung der Kompetenzen

Abbau des Demokratiedefizits und Ausweitung der Handlungsstrukturen politischer Öffentlichkeit

Politk




\section{$\mathrm{C} \cdot \mathrm{A} \cdot \mathrm{P}$}

\section{Anmerkungen}

1) Vgl. Walkenhorst, Heiko, 1999: Europäischer Integrationsprozess und europäische Identität. Die politische Bedeutung eines sozialpsychologischen Konzepts. Baden-Baden, S. 160-207, 212ff.; Weigl, Michael, 2006: Europas Ringen mit sich selbst. Grundlagen einer europäischen Identitätspolitik. Gütersloh.

2) Im Rahmen dieses Beitrags wird kein Anspruch auf Vollständigkeit erhoben.

3) Vgl. dazu zusammenfassend Misch, Axel, 1996: Legitimation durch Parlamentarisierung?, Das Europäische Parlament und das Demokratiedefizit der EU. In: Zeitschrift für Politikwissenschaft. Jg. 6, Heft 4, S. 969-995; Höreth, Marcus, 1999: Die Europäische Union im Legitimationstrilemma. Zur Rechtfertigung des Regierens jenseits der Staatlichkeit. Baden-Baden, S. 42-52; Peters, Anne, 2001: Elemente einer Theorie der Verfassung Europas. Berlin, S. 662-670.

4) $\mathrm{Zu}$ den beiden Varianten des Demokratiedefizits, die "Standardvariante“ und die „substanzielle Variante", vgl. Grande, Edgar, 1996: Demokratische Legitimation und europäische Integration. In: Leviathan. Jg. 24, Heft 3, S. 341-346.

5) Vgl. dazu Grande (Anm. 4); Höreth (Anm. 3), S. 52-64; Grimm, Dieter, 1995: Braucht Europa eine Verfassung? In: Juristenzeitung. Jg. 50, Heft 12, S. 581-591; Kielmannsegg, Peter Graf, 1996: Integration und Demokratie. In: Markus Jachtenfuchs/Beate Kohler-Koch (Hrsg.): Europäische Integration. Opladen, S. 47-71. 6) Vgl. dazu und zum Folgenden Hurrelmann, Achim, 2005:Verfassung und Integration in Europa. Wege zu einer supranationalen Demokratie. Frankfurt/Main, New York, S. 103-106.

7) Easton, David, 1965: A Systems Analysis of Political Life. New York, S. 267-277.

8) Schwaabe, Christian, 2005: Politische Identität und Öffentlichkeit in der Europäischen Union. Zur Bedeutung der Identitätsdiskurse im „post-abendländischen“ Europa. In: Zeitschrift für Politik. Jg. 52, Heft 4, S. 421-447, hier S. 423f.

Dagegen für eine Legitimation der EU durch effiziente Aufgabenerfüllung ohne die Notwendigkeit an zusätzlicher Input-Legitimation insbesondere Majone, Giandomenico, 1999: The Regulatory State and its Legitimacy Problems. In: West European Politics. Jg. 22, Heft 1, S. 1-24; Majone, Giandomenico, 1998: Europe's"Democratic Deficit”: The Question of Standards. In: European Law Journal. Jg. 4, Heft 1, S. 5-28; Scharpf, Fritz W., 2004: Legitimationskonzepte jenseits des Nationalstaats. Max-Planck-Institut für Gesellschaftsforschung, Working Paper Nr. 6. Köln; Scharpf, Fritz W., 1999: Regieren in Europa: Effektiv und demokratisch? Frankfurt/Main; Scharpf, Fritz W., 1998: Demokratie in der transnationalen Politik. In: Wolfgang Streeck (Hrsg.): Internationale Wirtschaft, nationale Demokratie: Herausforderungen für die Demokratietheorie. Frankfurt/Main, S. 151-174; Scharpf, Fritz W., 1998: Demokratische Politik in der internationalisierten Ökonomie. In: Michael Th. Greven (Hrsg.): Demokratie - eine Kultur des Westens? Opladen, S. 81-103.

9) Vgl. Europäische Kommission: Eurobarometer. Die öffentliche Meinung in der Europäischen Union Bericht Nr. 64, Dezember 2005, und Nr. 63, Juli 2005, abrufbar unter http://ec.europa.eu/ public_opinion/index_en.htm: 43/47 Prozent der EU-Bürger erachten den Kampf gegen Arbeitslosigkeit und 44/44 Prozent gegen Armut und soziale Ausgrenzung als vordringlichste Aufgabe der EU. Freilich fallen die Erwartungen in den einzelnen Mitgliedsländern zum Teil sehr unterschiedlich aus.

10) In den folgenden Ausführungen des Beitrags bleibt die Stärkung der Output-Legitimität der EU unberücksichtigt.

11) Hix, Simon, 2006: Why the EU needs (Left-Right) Politics? Policy Reform and Accountability are Impossible without it. In: Simon Hix/Stefano Bartolini: Politics: The Right or the Wrong Sort of Medicine fort he EU? Notre Europe, Policy Paper No. 19, S. 1-28, hier S. 3 f.

Für ein gleichberechtigtes Zusammenspiel von Output- und Input-Legitimität ebenfalls Rumler-Korinek, Elisabeth, 2003: Kann die Europäische Union demokratisch ausgestaltet werden? In: Europarecht. Jg. 38, Heft 2, S. 327-342; Schäfer, Armin, 2006: Die demokratische Grenze output-orientierter Legitimation. In: Integration. Jg. 29, Heft 3, S. 187-200.

12) Zur Existenz einer kollektiven Identität als Bedingung für die faktische Akzeptanz einer politischen Ordnung und damit deren diffuser Unterstützung vgl. Hurrelmann (Anm. 6), S. 122-131.

13) Vgl. zum Folgenden auch Thalmaier, Bettina, 2005: Braucht die EU eine eigene Identität? In: Helmut Heit (Hrsg.): Die Werte Europas. Verfassungspatriotismus und Wertegemeinschaft in der EU? Münster, S. 215-230.

14) Das traditionelle Integrationsziel „Europäischer Bundesstaat" wird ebenso wie eine „Europäische Konföderation“ aus analytischen und normativen Gründen abgelehnt, vgl. dazu Thalmaier, Bettina, 2005: Die zukünftige Gestalt der Europäischen Union - Integrationstheoretische Hintergründe und Perspektiven einer Reform. Baden-Baden, S. 223ff.

15) Jachtenfuchs, Markus/Beate Kohler-Koch, 1995: Regieren im dynamischen Mehrebenensystem. Mannheimer Zentrum für Europäische Sozialforschung, Arbeitspapiere Arbeitsbereich III/12, Mannheim, S. 6. 
16) Vgl. Utzinger, André, 2005: Mythen oder Institutionen? Zur Bildung kollektiver Identitäten im postnationalen Europa. In: Francis Cheneval (Hrsg.): Legitimationsgrundlagen der Europäischen Union. Münster, S. 235-251; Giesen, Bernhard, 1999: Kollektive Identität. Die Intellektuellen und die Nation 2. Frankfurt/Main.

17) Vgl. Jachtenfuchs, Markus, 1997: Die Europäische Union - ein Gebilde sui generis? In: Klaus Dieter Wolf (Hrsg.): Projekt Europa im Übergang. Probleme, Modelle und Strategien des Regierens in der Europäischen Union. Baden-Baden, S. 15-35; Kohler-Koch, Beate/Markus Jachtenfuchs, 1996: Regieren in der Europäischen Union - Fragestellungen für eine interdisziplinäre Europaforschung. In: Politische Vierteljahresschrift. Jg. 37, Heft 3, S. 537-556.

18) Walkenhorst (Anm. 1), S. 135.

19) Vgl. Jachtenfuchs/Kohler-Koch (Anm. 17).

20) Gerhards, Jürgen, 2003: Identifikation mit Europa. Einige begriffliche Vormerkungen. In: Jutta Allmendinger (Hrsg.): Entstaatlichung und soziale Sicherheit. Verhandlungen des 31. Kongresses der Deutschen Gesellschaft für Soziologie in Leipzig 2002, Teil 1. Opladen, S. 467-474, hier S. 471.

21) Vgl. Schneider, Heinrich, 1998: Zukunftsperspektiven der Europäischen Union. In: Waldemar Hummer (Hrsg.): Die Europäische Union nach dem Vertrag von Amsterdam. Wien, S. 331-382; Schmuck, Otto, 2001: Die Diskussion um die europäische Verfassung. In: Zeitschrift für Politikwissenschaft, Jg. 11, Heft 1, S. 105124.

22) Vgl. Mähring, Matthias, 1998: Das Transparenzdefizit der Europäischen Union. In: Staatswissenschaften und Staatspraxis. Jg. 9, Heft 3, S. 315-342; Sobotta, Christoph, 2000: Transparenz in den Rechtsetzungsverfahren der Europäischen Union. Stand und Perspektiven des Gemeinschaftsrechts unter besonderer Berücksichtigung des Grundrechts auf Zugang zu Informationen. Baden-Baden.

23) Vgl. Grande, Edgar, 1995: Regieren in verflochtenen Verhandlungssystemen. In: Renate Mayntz/Fritz W. Scharpf (Hrsg.): Gesellschaftliche Selbstregelung und politische Steuerung. Frankfurt/Main u.a., S. 327368; Grande, Edgar, 2000: Multi-Level Governance: Institutionelle Besonderheiten und Funktionsbedingungen des europäischen Mehrebenesystems. In: Edgar Grande/Markus Jachtenfuchs (Hrsg.): Wie problemlösungsfähig ist die EU? Regieren im europäischen Mehrebenensystem. Baden-Baden, S. 11-30; Benz, Arthur, 1998: Politikverflechtung ohne Politikverflechtungsfalle - Koordination und Strukturdynamik im europäischen Mehrebenensystem. In: Politische Vierteljahresschrift. 39. Jg., Heft 3, S. 558-589; Scharpf, Fritz W., 1985: Die Politikverflechtungsfalle: Europäische Integration und deutscher Föderalismus im Vergleich. In: Politische Vierteljahresschrift. Jg. 26, Heft 4, S. 323-356.

24) Lepsius, M. Rainer, 1997: Bildet sich eine kulturelle Identität in der Europäischen Union? In: Blätter für deutsche und internationale Politik. Jg. 42, Heft 8, S. 948-955, hier S. 952.

25) Vgl. Walkenhorst (Anm. 1), S. 120-133.

26) Vgl. Moravcsik, Andrew, 1993: Preferences and Power in the European Union. A Liberal Intergouvernemental Approach. In: Journal of Common Market Studies. Jg. 31, Heft 4, S. 437-524.

27) Kaufmann, Marcel, 1997: Europäische Integration und Demokratieprinzip. Baden-Baden, S. 205.

28) Vgl. Walkenhorst (Anm. 1), S. 83-96.

29) Walkenhorst (Anm. 1), S. 142.

30) Weidenfeld, Werner/Janis A. Emmanouilidis et al., 2006: Die strategischen Antworten Europas. Centrum für angewandte Politikforschung, CAP-Analyse Nr. 4. München, S. 19.

31) Vgl. Hurrelmann (Anm. 6), S. 153, 156; Thalmaier (Anm. 14), S. 337-353.

32) Vgl. Kohler-Koch, Beate/Thomas Conzelmann et al., 2004: Europäische Integration - Europäisches Regieren. Wiesbaden, S. 195-200; Follesdal, Andreas/Simon Hix, 2005: Why There is a democratic Deficit in the EU: A Response to Majone and Moravcsik. European Governance Papers No. C-05-02, http://www.connnex-network.org/eurogov/pdf/egp-connex-C-05-02.pdf.

33) Die Ansicht, ein Demokratiedefizit existiere gar nicht, vertreten hingegen die Vertreter der Ansicht "Legitimation der EU durch effiziente Aufgabenerfüllung" (vgl. Anm. 8) sowie Autoren, die einen eigenständigen Legitimationsbedarf der EU ablehnen, weil diese mittelbar über die Mitgliedstaaten demokratisch legitimiert sei, insbesondere Moravcsik, Andrew, 2002: In Defense of the "Democratic Deficit": Reassessing the Legitimacy of the European Union. In: Journal of Common Market Studies. Jg. 40, Heft 4, S. 603-634; Moravcsik, Andrew, 2004: Is there a "Democratic Deficit“ in World Politics? A Framework for Analysis. In: Government and Opposition. Jg. 39, Heft 2, S. 336-363; Bundesverfassungsgericht, 1993: Urteil vom 12.10.1993. In: Neue Juristische Wochenschrift. H. 47, S. 3047-3058; Kaufmann (Anm. 27), mit weiteren Nachweisen.

34) Vgl. Europäische Kommission: Eurobarometer. Die öffentliche Meinung in der Europäischen Union Bericht Nr. 64, Dezember 2005, abrufbar unter http://ec.europa.eu/public_opinion/index_en.htm. Im Bericht Nr. 63, Juli 2005, waren es noch 53 Prozent.

35) Kohler-Koch/Conzelmann et al. (Anm. 32), S. 221.

36) Vgl. Kantner, Cathleen, 2004: Kein modernes Babel. Kommunikative Voraussetzungen europäischer Öffentlichkeit. Wiesbaden, S. 61-110. 


\section{$\mathrm{C} \cdot \mathrm{A} \cdot \mathrm{P}$}

37) Kielmannsegg (Anm. 5); Grimm (Anm. 5); Habermas, Jürgen, 1996: Braucht Europa eine Verfassung? Eine Bemerkung zu Dieter Grimm. In: Ders.: Die Einbeziehung des Anderen, Frankfurt/Main, S. 185-191; Gerhards, Jürgen, 2002: Das Öffentlichkeitsdefizit der EU im Horizont normativer Öffentlichkeitstheorien. In: Hartmut Kaelble/Martin Kirsch et al. (Hrsg.): Transnationale Öffentlichkeiten und Identitäten im 20. Jahrhundert. Frankfurt/Main, S. 135-158, hier S. 154; Eder, Klaus/Kai-Uwe Hellmann et al., 1998: Regieren in Europa jenseits öffentlicher Legitimation? Eine Untersuchung zur Rolle von politischer Öffentlichkeit in Europa. In: Beate Kohler-Koch (Hrsg.): Regieren in entgrenzten Räumen. Opladen, S. 321-344, hier S. 321. 38) Neuere empirische Studien legen diese Annahme nahe und widersprechen der pauschalen Annahme eines Öffentlichkeitsdefizits, vgl. Risse, Thomas, 2004: Auf dem Weg zu einer europäischen Kommunikationsgemeinschaft: Theoretische Überlegungen und empirische Evidenz. In: Claudio Franzius/Ulrich K. Preuß (Hrsg.): Europäische Öffentlichkeit. Baden-Baden, S. 139-153; Risse, Thomas, 2002: Zur Debatte um die (Nicht-)Existenz einer europäischen Öffentlichkeit. Was wir wissen und wie es zu interpretieren ist. In: Berliner Debatte Initial. Jg. 13, Heft 5/6, S. 15-23. Einen ausführlichen Überblick über den empirischen Forschungsstand gibt Kantner (Anm. 36), S. 130-162. Aktuell auch Tresch, Anke/Margit Jochum, 2005: Europäisierung der Öffentlichkeit als Legitimationsbedingung der EU. In: Francis Cheneval (Hrsg.): Legitimationsgrundlagen der Europäischen Union. Münster, S. 375-392; Trenz, Hans-Jörg (2005): Europa in den Medien. Die europäische Integration im Spiegel nationaler Öffentlichkeit. Frankfurt/Main, New York.

39) Fuchs, Dieter, 2000: Demos und Nation in der Europäischen Union. In: Hans-Dieter Klingemann/Friedhelm Neidhardt (Hrsg.): Zur Zukunft der Demokratie. Herausforderungen im Zeitalter der Globalisierung. Berlin, S. 215-236, hier S. 232.

40) Gerhards, Jürgen, 1993: Westeuropäische Integration und die Schwierigkeiten der Entstehung einer europäischen Öffentlichkeit. In: Zeitschrift für Soziologie. Jg. 22, Heft 2, S. 96-110, hier S. 98.

41) Schwaabe (Anm. 8), hier S. 441f.; Meyer, Thomas, 2004: Die Identität Europas - Der EU eine Seele? Frankfurt/Main, S. 56.

42) Auf andere diskutierte Gründe für das Öffentlichkeitsdefizit wie insbesondere die mangelnde Transparenz des europäischen Entscheidungsprozesses, die Sprachenvielfalt in der EU oder das Fehlen gemeinsamer europäischer Medien wird hier nicht eingegangen.

43) Meyer (Anm. 41), S. 171; Neidhardt, Friedhelm/Ruud Koopmanns et al., 2000: Konstitutionsbedingungen politischer Öffentlichkeit: Der Fall Europa. In: Hans-Dieter Klingemann/Friedhelm Neidhardt (Hrsg.): Zur Zukunft der Demokratie. Herausforderungen im Zeitalter der Globalisierung. Berlin, S. 263-293, hier S. 285f.

44) Zur EU als Konkordanzsystem vgl. Schmidt, Manfred G., 2000: Der konsoziative Staat. Hypothesen zur politischen Struktur und zum politischen Leistungsprofil der Europäischen Union. In: Edgar Grande/Markus Jachtenfuchs (Hrsg.): Wie problemlösungsfähig ist die EU? Regieren im europäischen Mehrebenensystem. Baden-Baden, S. 33-58; Peters, Anne, 2003: A Plea for a European Semi-Parliamentary and Semi-Consociational Democracy, European Integration online Papers. Jg. 7, Heft 3, http://eiop.or.at/eiop/ texte/2003-003a.htm; Costa, Oliver/Paul Magnette, 2003: The European Union as a Consociation? In: West European Politics. Jg. 26, Heft 3, S. 1-18.

45) Zwar ist der Anwendungsbereich von Mehrheitsentscheidungen im Rat, dem wichtigsten Entscheidungsgremium, über die Jahre kontinuierlich ausgeweitet worden, gleichwohl wird davon in der Praxis kaum Gebrauch gemacht, vgl. Maurer, Andreas, 2003: Auf dem Weg zur Staatenkammer. Die Reform des Ministerrates der EU. Stiftung Wissenschaft und Politik, SWP-Studie 6. Berlin, S. 30.

46) Vgl. dazu und zum Folgenden Meyer (Anm. 41), S. 61f.

47) Neidhardt/Koopmanns et al. (Anm. 43), hier S. 285.

48) Vgl. zur Interaktion der drei Akteursgruppen Sprecher, Medien und Publikum ausführlich Neidhardt/Koopmanns et al. (Anm. 43), hier S. 275-288; Gerhards (Anm. 37), hier S. 150-154.

49) Trenz, Hans-Jörg, 2006: Europäische Öffentlichkeit und die verspätete Politisierung der EU. In: Internationale Politik und Gesellschaft. Heft 1, S. 117-133, hier S. 117.

50) Trenz (Anm. 49), hier S. 126.

51) Risse (Anm. 38), hier S. 146.

52) Vgl. Jopp, Mathias/Gesa-S. Kuhle, 2005: Wege aus der Verfassungskrise - die EU nach den gescheiterten Referenden in Frankreich und den Niederlanden. In: Integration. Jg. 28, Heft 3, S. 257-261.

53) Vgl. Thalmaier (Anm. 14), S. 133ff., 150ff.

54) Vgl. dazu ausführlich Thalmaier (Anm. 14), S. 189f., 379-382 mit weiteren Nachweisen, insbesondere Peters (Anm. 3), S. 758f.; Grande, Edgar, 1997: Post-nationale Demokratie - Ein Ausweg aus der Globalisierungsfalle? Institut für Sozialwissenschaften, Working Paper Nr. 2. München, S. 10-13.

55) Merkel, Wolfgang, 1999: Legitimitätsüberlegungen zu einem unionsspezifischen Demokratiemodell. In: Claus Giering/Josef Janning/Wolfgang Merkel/Michael Stabenow (Hrsg.): Demokratie und Interessenausgleich in der Europäischen Union. Gütersloh, S. 27-37, hier S. $33 f$. 


\section{$\mathrm{C} \cdot \mathrm{A} \cdot \mathrm{P}$}

56) Scharpf, Fritz W., 1994: Autonomieschonend und gemeinschaftsverträglich: Zur Logik einer europäischen Mehrebenenpolitik. In: Fritz W. Scharpf: Optionen des Föderalismus in Deutschland und Europa. Frankfurt/Main, S. 131-155, hier S. 133.

57) Vgl. Fischer, Thomas/Nicole Schley, 1999: Europa föderal organisieren. Ein neues Kompetenz- und Vertragsgefüge für die Europäische Union. Bonn, S. 79-82.

58) Vgl. dazu ausführlich Thalmaier (Anm. 14), S. 297f., 369-375 mit weiteren Nachweisen.

59) Vgl. dazu und zum Folgenden Mayer, Franz C., 2001: Die drei Dimensionen der Europäischen Kompetenzdebatte. In: Zeitschrift für ausländisches öffentliches Recht und Völkerrecht. Jg. 61, Heft 2-3, S. 576-638, hier insbesondere S. 611f., 597f.; Hetmeier, Heinz/Andrea V. Richter, 2001: Kompetenzabgrenzung in der Europäischen Union. In: Zeitschrift für Gesetzgebung, Jg. 16, Heft 4, S. 295-327, hier S. 305-308; Magiera, Siegfried, 2002: Zur Kompetenzneuordnung zwischen Europäischer Union und den Mitgliedstaaten. In: Integration. Jg. 25, Heft 4, S. 269-284, hier S. 274-277.

60) Peters (Anm. 3), S. 711.

61) Vgl. Westle, Bettina, 2003: Universalismus oder Abgrenzung als Komponente der Identifikation mit der Europäischen Union. In: Frank Brettschneider/Jan van Deth et al. (Hrsg.): Europäische Integration in der öffentlichen Meinung. Opladen, S. 115-152, hier S. 120f.; Kohli, Martin, 2002: Die Entstehung einer europäischen Identität: Konflikte und Potentiale. In: Hartmut Kaelble / Martin Kirsch et al. (Hrsg.): Transnationale Öffentlichkeiten und Identitäten im 20. Jahrhundert. Frankfurt/Main, S. 111-134, hier S. 125. 62) Zur Sozialpolitik siehe die Ausführungen im nächsten Abschnitt.

63) Vgl. dazu und zum Folgenden Beierwaltes, Andreas, 2000: Demokratie und Medien. Der Begriff der Öffentlichkeit und seine Bedeutung für die Demokratie in Europa. Baden-Baden, S. 241f.

64) Vgl. Beck, Ulrich/Edgar Grande (2004): Das kosmopolitische Europa. Frankfurt/Main, S. 164-168.

65) Beck/Grande (Anm. 64), S. 165.

66) Weidenfeld/Emmanouilidis et al. (Anm. 30), S. 8.

67) Vgl. Europäische Kommission: Eurobarometer. Die öffentliche Meinung in der Europäischen Union Bericht Nr. 64, Dezember 2005, und Nr. 63, Juli 2005, abrufbar unter http://ec.europa.eu/public_opinion/ index_en.htm: 77/77 Prozent der EU-Bürger sind für eine gemeinsame Sicherheits- und Verteidigungspolitik und 68/67 Prozent für eine gemeinsame Außenpolitik.

68) Siehe hierzu auch Weidenfeld/Emmanouilidis et al. (Anm. 30), S. 23.

69) Vgl. dazu die ausführliche Übersicht bei Janowski, Cordula, 2005: Demokratie in der EU gemäß der Europäischen Verfassung: parlamentarisch, post-parlamentarisch oder beides? In: Zeitschrift für Politikwissenschaft. Jg. 15, Heft 3, S. 793-824, hier S. 796-808.

70) So z.B. Holzinger, Katharina/Christoph Knill, 2001: Institutionelle Entwicklungspfade im Europäischen Integrationsprozess: Eine konstruktive Kritik an Joschka Fischers Reformvorschlägen. In: Zeitschrift für Politikwissenschaft. Jg. 15, Heft 4, S. 987-1010; Emmanouilidis, Janis A., 2005: Overcoming the Constitutional Crisis. München.

71) Vgl. dazu ausführlich Thalmaier (Anm. 14), S. 353-364 mit weiteren Hinweisen. Ebenso Hix, Simon, 2003: Parteien, Wahlen und Demokratie in der EU. In: Markus Jachtenfuchs/Beate Kohler-Koch (Hrsg.): Europäische Integration. 2. Auflage, Wiesbaden, S. 151-180, hier S. 174-176; Janowski, Cordula, 2004: Die nationalen Parlamente und ihre Europa-Gremien. Legitimationsgarant der EU? Baden-Baden, S. 234-239, 250; Decker, Frank, 2003: Parlamentarisch, präsidentiell oder semi-präsidentiell? In: Aus Politik und Zeitgeschichte. Heft 1-2, S. 16-23, hier S. 20f.; Decker, Frank, 2000: Demokratie und Demokratisierung jenseits des Nationalstaates: Das Beispiel der Europäischen Union. In: Zeitschrift für Politikwissenschaft. Jg. 10, Heft 2, S. 585-629, hier S. 616-619.

72) Vgl. dazu auch die Ausführungen in 3.4.

73) Für europaweite Referenden beispielsweise Beck/Grande (Anm. 64), S. 352f.; Zürn, Michael, 1996: Über den Staat und die Demokratie im europäischen Mehrebenensystem. In: Politische Vierteljahresschrift. Jg. 37, Heft 1, S. 27-55, hier S. 49f.; Merkel (Anm. 55), hier S. 32f.; Abromeit, Heidrun, 1998: Ein Vorschlag zur Demokratisierung des europäischen Entscheidungssystems. In: PolitischeVierteljahresschrift, Jg. 39, Heft 1, S. 80-90.

74) Danach können mindestens eine Million Bürger aus einer „erheblichen Anzahl von Mitgliedstaaten die Kommission zu Vorschlägen für bestimmte Rechtsakte auffordern“. Die näheren Einzelheiten bleiben einem Europäischen Gesetz vorbehalten.

75) Vgl. Pernice, Ingolf, 2001: The Role of National Parliaments in the European Union. Walter-HallsteinInstitut für Europäisches Verfassungsrecht, Paper No. 5. Berlin; Huber, Peter M., 2001: Die Rolle der nationalen Parlamente bei der Rechtssetzung der Europäischen Union. Zur Sicherung und zum Ausbau der Mitwirkungsrechte des Deutschen Bundestages. Hans-Seidel-Stiftung, Aktuelle Analysen Nr. 24. München.

76) Vgl. Maurer, Andreas, 2004: Die nationalen Parlamente im Europäischen Verfassungsvertrag. Anforderungen für eine proaktive Ausgestaltung der Parlamente. Stiftung Wissenschaft und Politik, Diskussionspapier. Berlin. 


\section{$\mathrm{C} \cdot \mathrm{A} \cdot \mathrm{P}$}

77) Ebenso Fuchs (Anm. 39), hier S. 232; Meyer (Anm. 41), S. 169-175.

78) Gerhards (Anm. 40), hier S. 108.

79) Hurrelmann, Achim, 2002: Verfassungspolitik als Konstruktion von Lernprozessen? Konstitutionalisierung und Identitätsbildung in der Europäischen Union. Mannheimer Zentrum für Europäische Sozialforschung, Arbeitspapiere Nr. 51. Mannheim, S. 15.

80) Vgl. dazu und zum folgenden Gerhards (Anm. 40), hier S. 108.

81) $\mathrm{Zu}$ den Gründen vgl. die Nachweise in Anm. 71.

82) Piepenschneider, Melanie, 2004: Die Rolle der europäischen Parteien. In: Claudio Franzius/Ulrich K. Preuß (Hrsg.): Europäische Öffentlichkeit. Baden-Baden, S. 237-246, hier S. 245; Wolf, Sebastian, 2000: Ein Vorschlag zur Beseitigung von Repräsentations- und Legitimationsdefiziten in Rat und Europäischem Parlament. Überlegungen zur Debatte über die institutionelle Reform der EU. In: Politische Vierteljahresschrift. Jg. 41, Heft 4, S. 730-741, hier S. 736-740.

83) Vgl. Piepenschneider (Anm. 82).

84) So z.B. Meyer (Anm. 41), S. 170.

85) Vgl. dazu und zum Folgenden Peters (Anm. 3), S. 697f.; Grande (Anm. 54), S. 15.

86) Meyer (Anm. 41), S. 170.

87) Vgl. Kurpas, Sebastian/Michael Brüggemann et al., 2006: Mapping a Way to a European Public Sphere. Centre für European Policy Studies, CEPS Policy Brief, No. 101. Brussels.

88) Kurpas, Sebastian/Christoph Meyer et al., 2004: After the European Elections, before the Constitutions Referenda: Can the EU Communicate Better? Centre für European Policy Studies, CEPS Policy Brief, No. 55. Brussels.

89) Zu den nachfolgenden Vorschlägen vgl. insbesondere European Policy Institutes Network (2005): A Citizens Compact: Reaching out to the Citizens of Europe. Working Paper No. 14, Brussels.

90) Hurrelmann (Anm. 6), S. 156.

91) Follesdal/Hix (Anm. 32); Hix (Anm. 11).

92) Risse (Anm. 38), hier S. $250 f$. 



\section{$\mathrm{C} \cdot \mathrm{A} \cdot \mathrm{P}$}

$\mathrm{C} \cdot \mathrm{A} \cdot \mathrm{P}$

Centrum für angewandte

Politikforschung

(C) 2006

Maria-Theresia-Str. 21

81675 München

Telefon $089 \cdot 21801300$

Telefax $089 \cdot 21801320$

E-Mail redaktion@cap-Imu.de

www.cap.Imu.de 